Ann. Biol. anim., Bioch. Biophys. tg66, 6 (1), r3-32.

\title{
LA CELLULE ÉPITHÉLIALE ABSORBANTE DE L'INTESTIN GRÊLE DU PORC ULTRASTRUCTURE
}

\author{
N. VODOVAR, J.-E. FLÉCHON \\ avec la collaboration technique de Marie-José Boufflers, Françoise Pessey et M. Niekerk \\ Station centrale de Nutrition et Station centrale de Physiologie animale \\ Centre national de Recherches zootechniques, 78 - Jouy-en-Josas
}

SOMMAIRE

La structure et la fonction de la cellule épithéliale absorbante de l'intestin grêle sont étroitement liées. De nombreuses techniques sont appliquées à l'étude des phénomèness de l'absorption du nutriment ingéré et, en particulier, de l'absorption des graisses ; cependant il est de toute évidence nécessaire, pour faire avancer l'état actuel de nos connaissances en ce domaine, de connaître l'organisation et l'ultrastructure des éléments cellulaires qui participent aux différents processus de cette absorption.

La cellule épithéliale du porcelet à la naissance diffère de celle prélevée sur les animaux plus âgés par une répartition dissemblable des éléments cytoplasmiques et par la présence d'inclusions lipoprotéiques avant toute ingestion d'aliments. Les transformations structurales (migration du noyau, répartition homogène des mitochondries, diminution du nombre des tubules de RE dans la zone apicale) sont très rapides et caractéristiques, pendant les $3^{6}$ premières heures; elles ne sont achevées que 3 jours environ après la naissance, ce qui correspond à un renouvellement complet de la couche épithéliale chez le Porc.

La division cellulaire est limitée aux cryptes de la muqueuse; les jeunes cellules se déplacent, de la base vers le sommet de la villosité, où elles se détachent et tombent dans la lumière intestinale ; cette migration s'effectue par glissement sur la membrane " basale ", qui sépare la couche épithéliale du reste de la paroi. Au cours de ces déplacements, la cellule semble s'adapter progressivement à la fonction d'absorption.

La section transversale de la cellule épithéliale diminue progressivement tandis que les microvillosités, dont le nombre reste constant, s'allongent, ce qui a pour conséquence d'augmenter la surface d'échange avec le milieu intestinal.

\section{IN'T'RODUC'TION}

La relation entre la structure et la fonction est évidente au niveau tissulaire. Elle l'est davantage encore au niveau cellulaire. L'étude raisonnée d'un phénomène biochimique, de ce fait, est difficilement concevable sans tenir compte de la structure des éléments cellulaires qui entrent en jeu. L'absorption des nutriments in- 
gérés, en général, et plus précisément des graisses alimentaires (substances insolubles dans l'eau), est étroitement liée à la structure des cellules épithéliales (CE) qui tapissent la villosité et bordent la lumière intestinale. Une étude rationnelle de ce phénomène ne pourrait donc être entreprise sans tenir compte de la structure particulière des éléments de la $\mathrm{CE}$, de sa maturation, de son renouvellement rapide. De nombreux chercheurs (que nous essaierons de passer en revue) ont étudié, suivant les moyens techniques dont ils disposaient, la cellule épithéliale de l'intestin grêle et notamment sa surface libre avec laquelle le nutriment ingéré vient d'abord en contact avant la pénétration.

Pour la première fois, HENI Ê a signalé, en I84I, que cette surface présentait une structure spéciale. GRUBY et DEI.AFOND (I843), étudiant la morphologie microscopique et la physiologie de la cellule épithéliale intestinale chez la plupart des espèces domestiques, ont observé, sur cette surface libre, des éléments qui ressemblaient à des corps vibratiles. A la suite de ces premières observations, KöLLIKER (I855), ainsi que FUNKE (I856), sur le Lapin, WeICKER (I857), sur le Chien, ont décrit la surface apicale de la cellule épithéliale intestinale comme une couche différenciée, pourvue de pores-canaux qui, pensaient-ils, constituaient les voies de pénétration des graisses. Pour BRETTAUER et STEINACH (I857), qui ont étudié cette cellule chez 1'Homme, le Cobaye, le Chien et le Lapin, il s'agissait d'éléments en forme de bâtonnets et non de pores-canaux.

Ces travaux, exécutés en même temps et souvent sur les mêmes espèces, ont abouti à des interprétations différentes de la structure et de la fonction de la surface libre de la cellule épithéliale intestinale; ils ont été à la base de nombreuses controverses qui ont duré près d'un siècle.

Pour Heidenhais (I888), la structure particulière de la couche apicale de la cellule épithéliale devait être une transformation protoplasmique, irrégulière et non permanente; ThanhofFer (I93I), pensait que cette structure de la couche apicale était un effet d'optique et que, histologiquement, il ne s'agissait ni de porescanaux ni de bâtonnets, ceci malgré les affirmations de ZIMMERMANN (I898), selon lesquelles il s'agissait bien de structures en forme de bâtonnets entourés par du cytoplasme.

Ces différentes thèses ont été passées en revue et conmentées par PATzEIT ( 1936$)$.

Les travaux de BAKER (I942, I95I), ont incontestablement marqué une étape dans la connaissance de la structure et du fonctionnement de la "bordure striée " ou " bordure en brosse ". Cependant, à cause du faible grossissement de la microscopie optique, ces travaux histologiques, ainsi que ceux qui les ont précédés, n'ont pu donner une image réelle de la structure de la bordure striée et nous confirmer tout ou partie des théories proposées.

Grâce à son haut pouvoir de résolution, le microscope électronique n'a cessé, depuis une quinzaine d'années, d'apporter des précisions sur la structure de la cellule épithéliale absorbante et de sa surface libre, que l'on sait maintenant constituée de "microvillosités" (mv).

GRANGER et BAKER (I949, I950) ont été les premiers à étudier les mv de la cellule intestinale du Rat au microscope électronique et à montrer que ces projections cytoplasmiques de forme cylindrique sont arrondies à leur extrémité, séparées les unes des autres et perpendiculaires à la surface de la cellule. Ces observations 
ont été confirmées et complétées par DaLTon et al. (I950), DaLTON (I95I), puis WEISS (I955 $a, b$ ).

Depuis lors au cours des dix dernières années, de nombreux travaux, effectués surtout sur le Rat, ont permis de mettre en évidence la complexité structurale de la CE de l'intestin grêle et, principalement, celle de sa région apicale dont le rôle dans le phénomène d'absorption est de première importance. Il a été montré que chaque cellule, entourée par la membrane plasmique, reste séparée de la cellule voisine par l'espace intercellulaire, excepté au niveau de la zone apicale (bandelette obturante) où les deux membranes sont en contact direct et assurent, avec les desmosomes et les interdigitations, l'intégrité de l'épithélium (SJÖSTRAND et ZETTERQVIST, I955; ZetTergvist, I956 ; Palay et KarLin, r959 ; SJöstrand, I963a).

L'épaisseur de la membrane plasmique, formée de 3 couches sur tout le pourtout de la CE, varie entre 80 et I $40 \AA$ stivant les auteurs et stivivant l'espèce étudiée (ZetTERQVist, I955; RobertSon, I959, I96I ; Sjöstrand, I963 $a, b, c$ ). Pour certains chercheurs, la membrane plasmique de la surface libre est légèrement plus épaisse que celle du reste de la cellule et diffère nettement des autres cytomembranes (Sjöstrand, I959, Ig63 $b, c, d$ ).

En traitant la muqueuse intestinale du Rat par une solution hypertonique de chlorure de sodium, à des temps variables avant la fixation, MILI_INGTON et FinEAN (I962, I963) ont précisé la structure des mv et, spécialement, celle de la membrane plasmique et des fibres des mv. I a mise en évidence des mv sur la surface libre de la $\mathrm{CE}$ a permis de constater une augmentation considérable de la surface absorbante. Cette augmentation étant fonction du nombre et des dimensions des mv (sur lesquelles les chercheurs ont fourni des données numériques variables) peut être néanmoins estimée à 20 fois environ:

L'évolution des mv, pendant la maturation de la CE, n'a pas été analysée.

La zone apicale dépourvue d'organites cellulaires n'a été que partiellement explorée. La relation de la membrane plasmique libre avec l'endoplasme, à travers cette couche, doit être précisée si 1'on cherche à expliquer l'acheminement des lipides à travers la $\mathrm{CE}$.

Il est de 1'opinion de la plupart des chercheurs que le RE est relativement peu abondant dans la $\mathrm{CE}$ (par rapport à une cellule du pancréas ou à un lymphocyte) et, en général, orienté parallèlement à son grand axe. Son rôle, singulièrement dans le transport des lipides, reste à déterminer. L'étude de l'appareil de Golgi, surtout en fonction du rôle qu'il pourrait jouer dans l'absorption des lipides, ne semble pas apporter de conclusions précises et concordantes.

Après l'étude histologique de la paroi de 1'intestin grêle du Porc (VoDovar, I964) et du lieu de l'absorption des graisses de différentes natures (Vodovar et al., I965), nous avons essayé d'apporter ici une contribution à la connaissance de l'ultrastructure de la CE; (noyau exclu) du Porc.

Notre attention s'est portée, en particulier, sur la structure de la membrane plasmique, de la microvillosité, du réticulum endoplasmique et de ses variations, suivant 1'âge de l'animal et la maturité de la cellule épithéliale. En effet, ces éléments nous paraissent jouer un rôle de premier ordre dans les problèmes de pénétration et de transport des graisses alimentaires à travers la cellule absorbante. 


\section{MATÉRIEL, ET MÉTHODES}

Les fragments de la paroi de l'intestin grêle sont prélevés dans la partie proximale du jéjunumiléon, sur des porcs d'âges différents, suivant la technicue décrite précédemment (Vodovar, 1964).

Pour suivre les modifications structurales normales de la cellule épithéliale pendant le temps de l'absorption et pendant le jeûne, le dernier repas est donné, suivant l'animal, entre I heure et I 8 heures, avant le prélèvement des tissus.

Les tissus prélevés sur des animaux à jeun depuis plus de I 8 heures présentaient des modifications cytoplasmiques irrégulières et variables et n'ont pas fait l'objet de cette étude.

Les fixations sont effectuées avec:

a) Le tétroxyde d'osmium à I p. Ioo, tampon véronal-acétate et à 2 p. 100, tampon phosphate de Milloning, pendant $\mathrm{I}$ heure.

b) Le permanganate de potassium à $0,6 \mathrm{p}$. 100 et à $2 \mathrm{p}$. 100 .

c) La glutaraldéhyde à 6 p. Ioo avec postfixation dans le tétroxyde d'osmium à 2 p. 1000 ou le permanganate de potassium à $\mathrm{I}$ p. $\mathbf{\text { I }} 0$.

Les fragments de tissus d'environ I $\mathrm{mm}^{3}$ sont inclus dans le méthacrylate prépolymérisé ou dans le styrène-1néthacrylate (KUSHIDA, 196r) et aussi le vestopal. Les coupes sont obtenues au microtome Porter-Blum à une épaisseur de 500 à I $000 \AA$. Les tissus sont constratés à l'acétate d'uranyle avant inclusion ou sur coupes.

Ies coupes, placées en sandwich entre un film de fornvar et de carbone dans le cas du méthacrylate, ont été observées avec l'Elmiscop I Siemens à un grossissement compris entre 3 ooo et 40000 .

Ces techniques nous ont permis d'obtenir à la fois une bonne préservation des tissus et des lipides et un contraste satisfaisant, ce qui est important pour notre étude parallèle de l'absorption.

Ont été étudiées successivement les cellules des parties basales, des parties intermédiaires et des sommets des villosités, chez des animaux d'âges différents (fig. 1).

\section{RÉSULTATS}

\section{A - Forme et dimensions de la cellule épithéliale du Porc (fig. B)}

Disposées en épithélium unistratifié bordant la lumière intestinale, les $\mathrm{CE}$, dont l'emplacement est identique sur les villosités, sont morphologiquement semblables chez des porcs de même âge. Ces cellules, qui tapissent la surface libre des villosités, peuvent être considérées comme les éléments absorbants des substances ingérées et notamment des graisses, sur toute la longueur de l'intestin grêle (Vodovar et al., Ig65).

Chez le nouveau-né, les $\mathrm{CE}$ sont approximativement cylindriques, puis leur section transversale devient polygonale (pentagonale ou hexagonale suivant le nombre des cellules adjacentes). Seule la surface apicale de ces cellules est libre. La section longitudinale est grossièrement rectangulaire; des irrégularités (espaces intercellulaires variables, plissements ou élargissements latéraux, fig. 2, I2) sont fréquentes sur les côtés et à la base.

Les dimensions de la CE varient suivant son emplacement sur la villosité, c'està-dire suivant son degré de maturité. En effet, la multiplication cellulaire se produit, sauf de rares exceptions, dans les cryptes; les jeunes cellules arrivent à maturité au cours de leur migration vers le sommet des villosités ; puis elles s'en détachent et tombent dans la lumière intestinale. La durée de vie d'une $\mathrm{CE}$ de la partie proxi- 
male du jéjunum-iléon, chez le Porc de 5 semaines, est de 1'ordre de 36 à 72 heures (VoDOvar, non publié).

La longueur des $\mathrm{CE}$; est la plus courte pour les cellules les plus jeunes et devient plus grande au fur et à mesure qu'elles se rapprochent du sommet; elle varie entre 25 et $5^{\circ} \mu$; la moyenne, pour la partie distale de la villosité est de $40 \mu$. Leur largeur est inversement proportionnelle à leur longueur : les cellules les plus jeunes ont donc le plus grand diamètre ; cette dimension est comprise entre 6 et I $2 \mu$, pour l'ensemble des cellules épithéliales.

La section transversale de la cellule n'est donc pas constante : la moyenne obtenue après de nombreuses mesures de cellules situées sur la partie distale des villosités et au niveau de la couche apicale est d'environ $45 \mu^{2}$; le volume semble rester constant du fait de la variation en sens inverse de la section et de la hauteur (ordre de grandeur $2000 \mu^{3}$ ).

L'âge de l'animal semble avoir peu d'influence sur les dimensions de la cellule.

\section{$\mathrm{B}$ - Membrane plasmique}

Le cytoplasme de chaque cellule est limité sur tout son pourtour, y compris les microvillosités, par une ligne opaque propre à chaque cellule : c'est la menbrame plasmique. Elle est formée des 3 couches classiques : une couche interne et une externe, opaques, limitant une zone intermédiaire plus claire. Les membranes contiguës des CE adjacentes sont séparées par un espace intercellulaire, excepté à la limite des surfaces libre et latérale.

La membrane plasmique de la surface libre, tant sur la microvillosité qu'au fond de l'espace intermicrovilleux, est d'une épaisseur totale d'environ II5 $\AA$ pour des coupes fixées au tétroxyde d'osmium. La couche opaque interne, plus dense que la couche externe, a une épaisseur régulière d'environ $35 \AA$. La couche opaque externe, moins dense, surtout chez les animaux sacrifiés au moment de la digestion, présente, au sommet des $\mathrm{mv}$, un aspect légèrement effiloché. Son épaisseur, sans être partout régulière, est d'environ $40 \AA$, ainsi que celle de la couche intermédiaire claire (fig. 7,8). On n'observe aucune différence chez les animaux restés à jeun jusqu'à I 8 heures et chez ceux nourris quelques heures avant le prélèvement.

Ce type de structure membranaire (constitué de 3 couches) est le plus courant; néanmoins, $\mathrm{n}^{n-1}$ avons parfois observé d'autres aspects : dans certains cas, la membrane semble alors formée, non plus de 3 couches parallèles, mais d'éléments sphériques qui se succèdent.

Sur coupes de tissus fixés au permanganate de potassium, les couches opaques sont plus nettement délimitées et la membrane plasmique apparaît légèrement plus épaisse.

L'épaisseur totale de la membrane plasmique latérale et basale de la cellule est de l'ordre de roo $\AA$; celle de la couche interne étant de $30 \AA$, la couche intermédiaire et la couche externe ont environ chacune $35 \AA$ d'épaisseur.

Le passage de la membrane plasmique de la surface libre à la membrane latérale, au niveau de la couche apicale, est caractérisé, à de rares exceptions près (fig. 4, 7), par la fusion des couches opaques externes des membranes plasmiques des 
cellules adjacentes. Ceci engendre une formation nommée " jonction cellulaire ", constituée de 5 couches successives, 3 opaques et 2 plus claires. Cette espèce de ceinture est disposée autour de la zone apicale de chaque cellule sur une hauteur de $0, I_{5}$ à $0,20 \mu$ environ. C'est la "bandelette obturante " ou "terminal bar ". On observe quelquefois l'absence de jonction cellulaire (fig. 5), surtout dans la partie distale des villosités. Ceci peut s'expliquer par l'écartement des cellules au passage des lymphocytes (souvent observés dans l'espace intercellulaire) ou par l'élimination de cellules à mucus ou encore par la chute des CE.

En dessous et sur tout le pourtour de la cellule, les membranes sont séparées par l'espace intercellulaire ; elles sont cependant pourvues d'éléments qui permettent de maintenir la cohésion et l'intégrité de la couche épithéliale. Environ 0,2 à $0,3 \mu$ en-dessous de la bandelette obturante, on trouve des dépôts intermittents de substance opaque (osmiophile), de part et d'autre de la même zone des deux cellules adjacentes (fig. 4, 5, 7, 9). L'espace intercellulaire, à ce niveau, est devenu plus large sur une longueur de quelques dixièmes de $\mu$. A partir de ces desmosomes, de nombreuses fibrilles rayonnent tout autour et surtout vers la couche apicale dans laquelle elles s'insèrent. Des structures desmosomiques semblables, mais dont les espaces intercellulaires sont moins élargis, se trouvent disséminées, sans ordre, sur tout le pourtour de la cellule; cependant ces différenciations membranaires entre cellules adjacentes sont plus abondantes sur la moitié supérieure de la cellule. Signalons rapidement (nous :reviendrons plus loin sur ce point) qu'à un niveau situé en dessous de la première rangée de desmosomes, la membrane plasmique semble, assez régulièrement, établir la liaison avec le réticulum endoplasmique (fig.7).

A partir de $0,4 \mu$ environ au-dessous de la surface libre de la cellule, les membranes plasmiques latérales forment des replis, variables en nombre et en aspect, soit parallèles au grand axe de la cellule, soit perpendiculaires à celui-ci. Ces nombreuses interdigitations permettent un maintien souple de la cohésion des CE (fig.2, I2, I3). La substance intercellulaire constitue peut-être aussi un facteur de cohésion des cellules épithéliales.

Entre la bandelette obturante et les desomosomes, la distance séparant les membranes de deux cellules adjacentes (espace intercellulaire) est assez régulière et d'environ Ioo $\AA$ pour des tissus prélevés sur des animaux soit à jeun, soit nourris juste avant le sacrifice. Au-dessous de la première tangée des desmosomes jusqu'à la basale, ces espacements sont irréguliers (fig. 7, I2, I3). Bien que d'aspect différent d'une coupe à l'autre, il ressort néanmoins que les espaces intercellulaires sont plus importants che $z$ le porcelet au moment de la naissance, puis chez les animaux abattus pendant l'absorption que chez ceux abattus à jeun. Les espaces les plus grands, dans lesquels se trouvent souvent des substances osmiophiles, sont situés au sommet des interdigitations et vers la base de la cellule (fig. 7, II, I2). Ces espaces ont une largeur très variable (de roo à 3 ooo $\AA$ environ).

La membrane cytoplasmique et la basale suivent, le plus souvent, un parcours rectiligne et parallèle (fig. II). La basale sépare la cellule des éléments du stroma (notamment de la lactéale centrale et de nombreux vaissaux capillaires sanguins). Chez le porcelet, à la naissance, elle est dense et de même opacité que la membrane plasmique (fig. 2) ; elle devient rapidement plus large et moins opaque. Les deux membranes sont séparées par une couche plus claire et d'une largeur assez constante, sauf exception (fig. 2, II). 


\section{C - Microvillosites de la cellule épithéliale (fig. C)}

Ces structures cylindriques, arrondies à l'extrêmité, constituent des prolongements du cytoplasme de la région apicale et se répartissent sur la surface libre de la $\mathrm{CE}$ du Porc; elles ont des dimensions uniformes pour chaque cellule, mais variables suivant que la cellule se trouve dans les cryptes ou sur les villosités. Dans ce dernier cas, il faut encore préciser qu'il s'agit de la partie proximale de la villosité (base de la villosité), de la partie médiane ou de la partie distale (sommet de la villosité) (fig. 6, 7,8,9). Ces mv sont assez régulièrement espacées (fig. Io), sans arrangement particulier, contrairement à ce qui a été signalé chez le Rat par PALAY et KARLIN (I959).

Les invaginations du fond des espaces intermicrovilleux sont très nombreuses chez le porcelet pendant les premières 36 heures après la naissance, qu'il soit nourri ou non, mais assez rares chez les animaux adultes ou en croissance; toutefois, elles semblent être plus nombreuses chez les animaux abattus après un jeûne prolongé.

\section{a) Nombre de mv par unité de surface.}

Déterminé sur des coupes parallèles à la surface de la cellule (fig. ro), ce nombre s'élève approximativement à 86 par $\mu^{2}$ dans la région distale de la villosité, à 58 par $\mu^{2}$ dans la région médiane où les variations sont plus importantes et à 37 par $\mu^{2}$ dans la région proximale. Nous avons vu que la section transversale de la cellule absorbante varie suivant les stades de la maturation : ainsi, dans la partie distale de la villosité, cette surface est en moyenne de $45 \mu^{2}$; le nombre des mv dans la même région étant de 86 par $\mu^{2}$, le nombre de mv par cellule épithéliale est approximativement de $3870(86 \times 45)$. Les cellules de la base, dont les côtés sont compris entre 8 et I $2 \mu$, ont une surface libre d'environ I0o $\mu^{2}$, ce qui donne un nombre de mv d'environ 3700 ( IOO $\times 37)$. On constate que le nombre des mv ne semble pas varier avec la maturité de la cellule (tabl. I).

\section{b) Longueur des $m$.}

Des résultats des mesures effectuées dans différentes régions et sur plusieurs animaux (tabl. I), il ressort que la moyenne des longueurs des mv sur les cellules de la région distale est de $I, 94 \mu$, les valeurs extrêmes étant I,6o et 2,35 $\mu$. Sur les côtés de la villosité (surface la plus importante), cette longueur est comprise entre 0,90 et $\mathrm{I}, 70 \mu$ avec une moyenne de $\mathrm{I}, 36 \mu$. A la base des villosités, la moyenne est de $0,96 \mu$ avec des écarts compris entre 0,80 et $I \mu$. La longueur des mv dans les pseudo-cryptes (parties de la villosité dont les surfaces sont tantôt libres, tantôt accolées) diffère peu de celle des mv de la base.

\section{c) Diamètre des $m v$.}

On constate que, pour les cellules des différentes régions de la villosité, ce diamètre croît en raison inverse de la hauteur des mv. Ainsi, les mv sont près de deux fois plus longues au sommet des villosités, mais leur diamètre est alors le plus faible (tabl. r).

Le diamètre moyen des mv est de $0,082 \mu$ pour la partie distale de la villosité, de $0,094 \mu$ pour la partie médiane et de 0, I Io $\mu$ pour la partie proximale. 
Le diamètre mesuré sur des coupes à différents niveaux entre la base et le sommet des mêmes microvillosités présente des différences négligeables.

d) Surfaces et volume des mv.

L'aire moyenne de la section transversale d'une mv sur les cellules situées au sommet des villosités est de $0,0053 \mu^{2}$, dans la partie médiane de $0,0069 \mu^{2}$ et à la base de $0,0095 \mu^{2}$. La surface cylindrique des mv est de $0,47 \mu^{2}$ au sommet de la villosité, de $0,38 \mu^{2}$ sur les côtés et de $0,33 \mu^{2}$ à la base (tab1. I).

\section{TABLEAU I}

Microzillosités (nombre, longueur, diamètre, coefficient d'augmentation de surface)

\begin{tabular}{|c|c|c|c|c|}
\hline & $\begin{array}{l}\text { Au sommet des } \\
\text { villosités }\end{array}$ & $\begin{array}{c}\text { Sur le côté des } \\
\text { villosités }\end{array}$ & $\begin{array}{l}\text { A la base des } \\
\text { villosités }\end{array}$ & $\begin{array}{l}\text { A la sortie des } \\
\text { cryptes }\end{array}$ \\
\hline Nombre de microvillosités par $\mu^{2}$ & 86 & 58 & 37 & 26 \\
\hline Nombre de nicrovillosités par cellule & 3870 & - & 3700 & - \\
\hline longueur d'ume microvillosité (en $\mu$ ) & $1,9 / 4$ & 1,36 & 0,96 & 0,82 \\
\hline Diametre d'une microvillosité (en $\mu$ ) & 0,082 & 0,091 & 0,110 & 0,130 \\
\hline $\begin{array}{c}\text { Surface de la section transversale } \\
\text { d'une microvillosité (en } \mu^{2} \text { ) }\end{array}$ & 0,00153 & $0,0065,5$ & 0,0095 & 0,0132 \\
\hline Surface d'une microvillosité (en $\mu^{2}$ ) & 0,17 & 0,38 & 0,33 & 0,33 \\
\hline Volume d'une microvillosité (en $\mu^{3}$ ) & 0,010 & 0,009 & 0,009 & 0,011 \\
\hline $\begin{array}{c}\text { Surface occupée par la section transver- } \\
\text { sale des microvillosités par } \mu^{2}\end{array}$ & 0,16 & $0,,^{\prime} \mathbf{t}^{\prime}$ & 0,35 & 0,34 \\
\hline Surface intermicrovilleuse par $\alpha^{2}$ & $0,5 \%$ & 0,60 & 0,65 & 0,66 \\
\hline $\begin{array}{c}\text { Coefficient d'augmentation de surface } \\
\text { libre }\left({ }^{1}\right)\end{array}$ & $\times 11$ & $\times \quad 2: 3$ & $\times 12$ & $\times 9$ \\
\hline
\end{tabular}

(1) Coefficient d'augmentation de surface libre :

Surface totale des microvillosités + surface intermicrovilleuse

Section transversale de la cellule au niveau apical

La différence de surface libre est relativement peu importante pour les mv des différentes régions. Il en est de même pour leur volume qui est de o,oro $\mu^{3}$ pour celles situées au sommet de la villosité et de $0,009 \mu^{3}$ pour celles de la partie intermédiaire et celles de la base.

e) Accroissement de la surface libre par les mv.

F́tant donné le nombre des mv par $\mu^{2}$, on trouve que l'ensemble des sections transversales des mv occupe $0,46 \mu^{2}$ dans la région distale, $0,40 \mu^{2}$ dans la région 
médiane et $0,35 \mu^{2}$ dans la région proximale, soit, suivant la région, 45 p. I00, 40 p. I0o et $35 \mathrm{p}$. IOO de la section transversale de la CE. La surface non occupée par les mv, ou surface intermicrovilleuse libre de la cellule, est de $0,54 \mu^{2}$ pour la partie distale, de $0,60 \mu^{2}$ pour les cellules de la région intermédiaire et de $0,65 \mu^{2}$ pour la partie proximale, ce qui correspond respectivement à $55 \mathrm{p}$. Ioo, $60 \mathrm{p}$. Ioo et $65 \mathrm{p}$. Ioo suivant les régions.

Par la présence des $\mathrm{mv}$, la surface libre de la CE absorbante est multipliée par un coefficient d'environ $4 \mathrm{I}$ dans la partie distale de la villosité, 23 dans la partie intermédiaire et I2 seulement pour les cellules de la base (tabl. I). L'aire membranaire libre (surface d'échange avec la lumière intestinale) s'élèverait à un maximum de l'ordre de $\mathrm{I} 700 \mu^{2}(45 \times 4 \mathrm{r})$ pour une cellule absorbante de la partie distale des villosités.

Signalons que peu de variations ont été observées en ce qui concerne le nombre et les dimensions des mv par rapport à 1'âge des animaux.

\section{$\mathrm{D}$ - Structure interne de la cellule épithéliale du Porc}

L,e volume total de la cellule épithéliale absorbante, quoique difficile à déter-miner, semble peu varier avec l'âge de l'animal et l'emplacement des cellules sur la villosité. (Il est évalué, très approximativement, à $2000 \mu^{3}$ ). Le rapport nucléoplasmique, en revanche, semble varier suivant l'âge et au cours de la migration des cellules entre la crypte et le sommet de la villosité. Le noyau, de forme sphérique et multilobé dans le jeune âge et dans les cellules immatures, occupe alors le tiers subapical de la cellule ; ensuite il devient ellipsoidal et se situe, la plupart du temps, dans la moitié basale de la cellule.

Si on l'observe à une haute résolution, on remarque que le cytoplasme est constitué par une substance fondamentale extrêmement fine, homogène, contenant des filaments et dans laquelle sont disséminés les éléments cytoplasmiques. Suivant la densité de la substance hyaloplasmique et suivant la répartition des éléments cytoplasmiques, plusieurs régions peuvent être distinguées sur une coupe parallèle au grand axe de la cellule.

a) Structure interne d'une microvillosité.

Quelle que soit la fixation utilisée, les mv apparaissent, en coupe transversale, formées de cytoplasme homogène pourvu d'un certain nombre de fibres rectilignes, groupées plus ou moins nettement autour du centre (fig. I0). Les fibres, en forme de baguettes, sont orientées de façon longitudinale et, au sommet des mv, semblent se prolonger jusqu'à la couche interne opaque de la membrane plasmique. A la base de la mv, le cytoplasme homogène se continue dans la zone apicale sans discontinuité, tandis que les fibres occupant la partie centrale de la mv traversent la couche apicale et se prolongent dans le cytoplasme sub-apical pour former un fuseau (fig. 5, 8). C'est ce qu'on appelle souvent les " racines " des mv; leur diamètre semble être uniforme (environ $50 \AA$ ).

b) Ectoplasme (zone apicale et périphérique du cytoplasme).

A partir de la base des mv et sous la membrane plasmique des espaces intermicrovilleux, s'étend la zone apicale dont l'épaisseur est d'environ 0, I $_{5}$ à $0,20 \mu$. 
Sa substance cytoplasmique, plus dense que dans les autres parties de la cellule, est riche en filaments orientés plus ou moins parallèlement à la surface de la cellule. Une zone cytoplasmique de même aspect, mais plus mince, peut être observée sur les faces latérale et basale de la cellule; c'est la couche ectoplasmique (PALAY et KARLIN, I959). On observe aussi, à ce niveau, entre les racines des mv, de nombreux tubules dont la paroi est formée d'une membrane cytoplasmique (Vovovar et Fléchon, I964; McNabB, I964) et qui, en cheminant à l'intérieur de cette couche, semblent relier le fond des espaces intermicrovilleux à un réseau de réticulum endoplasmique se trouvant à la limite inférieure de la zone apicale.

c) Endoplasme (cytoplasme compris entre l'ectoplasme et le noyau).

I)ans cette partie de la cellule, la substance fondamentale du cytoplasme, parsemée de granules ribosomiques, est moins dense et pourvue des éléments cytoplasmiques habituels (mitochondries, réticulum endoplasmique, etc.) et de structures ou d'inclusions occasionnelles. Ces éléments sont variables suivant 1'âge et la maturité de la $\mathrm{CE}$.

I $^{0}$ Les mitochondries, absentes de la zone apicale, sont disséminées dans le cytoplasme d'une façon différente suivant l'âge de l'animal et suivant la maturité de la CE. Chez le porcelet nouveau-né, les CE, qui ont la polarité renversée et ne sont pas encore fonctionnelles, ont leurs mitochondries placées presque exclusivement dans la région située au-dessous du noyau, celui-ci occupant la partie sub-apicale de la cellule (fig. I). Dès que l'animal absorbe la première nourriture, le noyau se déplace graduellement vers le centre, puis vers la partie basale de la CE; les mitochondries migrent sur les côtés, puis au-dessus du noyau. Quelque temps après, la répartition devient relativement égale dans toute la cellule. Trois jours après la naissance, pour les CE apicales occupant la partie distale des villosités, on compte 50 à 70 mitochondries par section parallèle au grand axe de la cellule, 25 à 35 par section perpendiculaire au grand axe, entre la zone apicale et le noyau; dans la partie située au-dessus du noyau, leur nombre est plus grand, le volume cytoplasmique étant supérieur ; mais, par unité de volume, il semble que la répartition doive être considérée comme homogène dans les cellules à maturité.

En coupe transversale, les mitochondries ont une section circulaire ou plus ou moins elliptique correspondant à des formes souvent cylindriques ou parfois sphériques; des ramifications ont été osbervées. Les dimensions des mitochondries ne sont pas constantes; le diamètre des sections circulaires varie entre 0,2 et $0,3 \mu$; la longueur des formes cylindriques atteint souvent $\mathrm{I}, 5 \mu$ et plus. Les mitochondries de forme cylindrique sont orientées, en majorité, suivant le grand axe de la cellule chez les jeunes animaux et dans les cellules au début de la maturation. Par la suite, on trouve autant de sections longitudinales que transversales sur une série de coupes. Pour l'ensemble du volume cytoplasmique, il semble que la forme, la taille et le nombre des mitochondries ne changent ni suivant l'âge de l'animal, ni suivant qu'il ait été à jeun ou non avant le sacrifice, ni suivant la maturité de la CE. Signalons enfin que les mitochondries ont une ultrastructure classique.

$2^{\circ}$ Le réticulum endoplasmique : 1'aspect et la quantité de réticulum endoplasmique contenu dans la cellule épithéliale absorbante du Porc est variable selon l'âge de l'animal, la maturité de la CE et le moment du prélèvement des tissus (soit sur un animal à jeun, soit après absorption de nutriment). 
Le réticulum endoplasinque granuleux $(R F R)$ est exceptionnellement présent dans la zone apicale. A la limite inférieure de cette zone, surtout chez le porcelet (VODOVAR, Fíf́chon, I964), il s'étend parallèlement à la surface libre de la cellule, pour entrer, nous semble-t-il, en rapport avec la membrane plasmique latérale à des niveaux plus ou moins éloignés au-dessous de la bandelette obturante (fig. 7). A partir de ce niveau, de nombreux réseaux de $\mathrm{RF} R$ cheminent dans la partie supranucléaire de la cellule, sur le côté et au-dessous du noyau (fig. 2, 3, 7, I2, I3). Ces formations d'aspect tubulaire, dont le diamètre interne varie entre roo et $25^{\circ} \AA$ orientées soit parallèlement au grand axe de la cellule, soit plus ou moins obliquement (fig. I2, I3), constituent un réseau aux fréquentes anastomoses et ramifications qui entoure les mitochondries et le noyau. Nombreux sont les cas où il semble que le RER s'unisse à la membrane plasmique latérale (fig. 7, I2, I3) (et ceci en particulier dans la région supra-nucléaire); il s'établirait ainsi une liaison entre l'intérieur de la cellule et l'espace intercellulaire.

La quantité de RER mise en évidence dans les tissus de très jeunes porcelets est bien supérieure à celle trouvée chez le porc adulte. Ein revanche, dans les cellules immatures et prélevées sur des animaux ayant subi un jê̂ne prolongé, le RE, $R$ semble moins abondant.

Le réticulum endoplasmique lisse (REL), S.L., comprend, d'une part, les cytomembranes qui forment l'appareil de Golgi, d'autre part, les autres cytomembranes qui constituent de nombreux canalicules et vésicules isolés et dépourvus de granulations.

L,e complexe de Golgi, passé en revue pat Dalton (I96I), a été étudié, pour le même type de cellules, chez d'autres espèces, par ZETTERqvist (I956), PALAY et KARLIN (I959) ; il se présente, dans les cellules épithéliales du Porc, sous forme d'amas de nombreuses vésicules, localisées généralement au-dessus du noyau ou, plus rarement, sur le côté. Leur nombre, variable suivant les cellules, apparaît également variable suivant l'incidence de la coupe d'une même cellule. Les membranes de l'appareil de Golgi, observées à une haute résolution, apparaissent également formées de 3 couches, d'une épaisseur totale de 65 à $75 \AA$, suivant que la fixation a été effectuée au tétroxyde d'osmium ou au permanganate de potassium. La forme de ces vésicules, circulaire ou aplatie, de diamètre extérieur variable, diffère peu suivant 1'âge de l'animal et la maturité de la cellule épithéliale absorbante ; mais les modifications sont importantes selon que les tissus ont été prélevés sur des animaux ayant subi un jeûne prolongé ou se trouvant au stade de l'absorption et, en particulier, de l'absorption des graisses. Dans ce dernier cas, ces vésicules sont remplies de substance osmiophile ; leur taille a augmenté et les espaces intervésiculaires semblent s'élargir.

Dans la zone apicale, le REIL est surtout abondant chez le jeune porcelet (fig. I,2). De nombreux canalicules et vésicules, très apparents à cet âge, semblent constituer un lien entre la membrane plasmique libre, pourvue d'invaginations, et l'endoplasme. Chez les animaux plus âgés, le cytoplasme de cette zone, devenu plus dense et plus complexe, complique la mise en évidence du REL. La manière dont ces canalicules confluent avec la membrane plasmique n'a pu être déterminée d'une façon précise.

Enfin, dans la couche sub-apicale, à tout âge, et aux différents stades de la maturité cellulaire, chez des animaux à jeun ou non, on trouve de nombreuses vésicules de diamètre variable, dépourvues de microsomes. 
La continuité entre le RER et le REI, semble pouvoir s'établir aussi bien dans la région golgienne que dans les autres parties du cytoplasme. Dans le cytoplasme sub-apical, le passage entre les deux types de réticulum se caractérise par un appauvrissement progressif en ribosomes jusqu'à leur complète disparition.

\section{DISCUSSION}

Les présentes observations montrent que les modifications structurales des $\mathrm{CE}$ s'effectuent, d'une part, pendant les quelques jours qui suivent la naissance, et, d'autre part, au cours de chaque cycle de renouvellement de la couche épithéliale et indépendamment de l'âge de l'animal.

Les premières modifications (migration du noyau vers la base, répartition homogène des mitochondries dans l'endoplasme, invagination de la membrane plasmique etc.) sont observées, en général, pendant les 3 premiers jours. Cependant les transformations les plus caractéristiques ont lieu surtout durant les 36 premières heures. Elles coïncident avec l'absorption du colostrum. Des constatations semblables ont été faites par MAtTison et KARLsson (I964). D'après ces auteurs, cette absorption se ferait par "pinocytose ", phénomène que nous n'avons observé qu'exceptionneilement.

\section{Maturation de la cellule épithéliale absorbante}

En comparant les CE des cryptes et de la base des villosités, lieu de leur multiplication (Leblond et Stevens, I948; Creamer, Shorter et Bamforth, I96I ; Vodovar, non publié, sur le Porc), aux cellules absorbantes qui tapissent les parties distales des villosités, on constate une inégalité importante dans leur différenciation. La maturation s'effectue donc au cours de la migration et nos observations concordent avec celles de TAyLOR et Adams'Tone (r964). Ainsi les cellules plus jeunes et moins adaptées à l'absorption se trouvent à la base des villosités, les plus âgées au sommet. La structure s'adapte progressivement à la fonction; ceci semble donner une explication à la question posée par FRAZER (I964) : pourquoi 1'intensité de l'absorption des graisses est-elle toujours plus grande au sommet des villosités?

Remarquons cependant que les délimitations dans les villosités, en zones de divisions cellulaires et en zones d'absorption, par exemple, ne sont ni très nettes, ni bien définies : il ne faut pas oublier que la muqueuse intestinale est en perpétuelle transformation (VODOVAR, r964) et que les différentes zones ne doivent pas être considérées comme permanentes et sans modifications.

\section{Membrane plasmique}

La membrane plasmique de la surface libre apparaît plus épaisse que la membrane plasmique latérale, quels que soient les moyens de fixation et d'inclusion. I'inégalité (II5 $\AA$ contre Ioo $\AA$ ) est comparable à celle observée par SJöSTRAND ) I963, b) chez la Souris ( $95 \AA$ et $82 \AA$ ). L'épaisseur de la membrane plasmique, chez 
le Porc, est égale à celle trouvée par Mili,ington et Finean (I962) chez le Rat. Les différences entre la membrane plasmique de la surface libre et la membrane plasmique latérale semblent s'inscrire en faux contre la thèse de " l'identité des membranes " (RoBERTSON, I957, I959, I96I).

Sur nos électromicrographies, la couche opaque externe est apparue toujours plus large et souvent moins dense que la couche interne ; cela correspond aux résultats publiés par MrLington et FinEAN, tandis que ceux de Sjöstrand sont inverses. Peut-on affirmer que l'inégalité d'épaisseur des deux couches opaques de la membrane donne une certitude à la théorie de SJös'rRAND (I963 c) (asymétrie de la membrane plasmique et différence de structure chimique des deux couches opaques)? La discontinuité occasionnelle de la membrane, déjà décrite par ZETTERQVIST (I956), et l'apparition d'éléments membranaires sphériques, signalée par SjösTRAND (I963 $c$ ) et par GRANEY (I964) constituent autant d'indications qui donnent à penser que ce problème de la structure membranaire n'est pas encore résolu. Il faut être prudent (FAVARGER, I959) et attendre la connaissance exacte de l'architecture moléculaire des différentes couches, avant de conclure avec assurance.

Les liens cellulaires et les espaces intercellulaires sont très variables d'une coupe à l'autre, suivant le niveau. Il n'est pas possible de parler de l'agencement régulier de ces espaces, comme cela a été dit pour certaines espèces (ZETTERQVIST, I956).

Il est intéressant de noter que la membrane plasmique ne semble varier ni avec l'âge de l'animal, ni avec la maturité de la cellule épithéliale absorbante, à la différence de la " basale " qui devient plus large et moins homogène après la première absorption.

\section{Microvillosités}

Présentes à tout âge des animaux et des cellules, les mv ne montrent pas une densité constante par unité de surface. Dans son travail, effectué sur l'homme, et qui est le seul, à ce jour, sur la question, BROWN (I962) avait émis l'hypothèse que l'augmentation de la densité des mv au cours de la maturation de la cellule était le résultat de la diminution de la surface de celle-ci, d'une part, et de la formation de nouvelles mv, d'autre part. Nos résultats numériques font penser que le nombre des microvillosités par cellule ne change pas au cours de la maturation de la cellule épithéliale et que, seule, la section transversale de cette dernière diminue, d'où une densité croissante par unité de surface. Ceci, à notre avis, est justifié, d'une part, par le nombre de mv relativement constant à tous les niveaux ( 3870 pour les cellules situées au sommet, 3700 pour celles situées à la base des villosités), d'autre part, par le fait que, aux divers stades de maturation, les mv sont toutes de la même taille pour une même cellule.

Le nombre des mv, donné par unité de surface ou par cellule, est variable suivant l'espèce et suivant les auteurs ; ainsi ZETTTERQVIST (I956) trouve $650 \mathrm{mv}$ par cellule de l'épithélium intestinal de Souris ; PALAY et KARL,IN (I959), pour la cellule du Rat, donnent le chiffre de I I25; quant à GRANGER et BAKER (I95I), pour la même espèce, ils trouvent $3000 \mathrm{mv}$ par cellule ; chez 1'Homme, AsrrworTH et al. (I96r) en trouvent I 8oo, HAUBRICH et al. (I959), 750. Ces résultats, bien qu'ils encadrent les nôtres, ne fournissent aucun moyen de comparaison; d'abord, ces auteurs n'ont pas tenu compte, en établissant ces chiffres, du lieu de prélèvement des cellules sur la villo- 
sité ; ensuite, ils ont pris pour la cellule épithéliale, quel que soit son emplacement sur la villosité et quelle que soit 1 'espèce considérée, une surface uniforme de I5 $\mu^{2}$.

La longueur des mv croît au cours de la maturation de la cellule. Les valeurs trouvées par Brown, chez 1'Homme, sont comprises entre $0,67 \mu$ et $\mathrm{I}, 36 \mu$. La comparaison avec nos résultats (tabl. I) permet de supposer qu'il peut exister une différence suivant les espèces.

Comme, au cours de la migration des cellules épithéliales, les mv s'allongent et que leur diamètre diminue, on constate que la surface et le volume de chaque mv (tabl. r) ne diffèrent que très légèrement pour les différentes zones des villosités. Remarquons l'analogie avec la cellule même, qui s'allonge au fur et à mesure de sa migration vers le sommet de la villosité, sans changement apparent de volume.

La détermination du nombre des mv et de la surface de leur section a été effectuée sur des électromicrographies de coupes dont la section est perpendiculaire, le plus possible, à l'axe des mv. C'est, nous semble-t-il, le seul moyen de faire une détermination exacte. Le nombre des mv obtenu, en se basant sur leur densité linéaire, sur des coupes parallèles à leur axe, nous a donné des résultats erronés, quelle que soit l'épaisseur de la coupe, Il en est de même pour le calcul du diamètre de section des mv. Toute comparaison des résultats avec ceux obtenus par la seconde méthode nous semble sans valeur. Suivant la zone des villosités sur laquelle se trouvent les cellules, les $\mathrm{mv}$ constituent un facteur variable d'augmentation de la surface libre par unité de surface de la cellule épithéliale absorbante (tabl. x). La valeur donnée par PALAY et KARLIN (I959) pour la cellule épithéliale du Rat, sans tenir compte de son emplacement, est de 24 .

Nous insistons sur le fait que, dans une population telle que celle représentée par les cellules épithéliales d'une villosité, et si l'on tient compte des variations et du changement de relief de la muqueuse intestinale, toute donnée numérique doit être interprétée avec prudence.

\section{Cytoplasme et éléments cytoplasmiques}

Les $\mathrm{mv}$ et la couche apicale constituent la partie du cytoplasme la plus différenciée de la $\mathrm{CE}$, absorbante, et, sans doute, la plus complexe du point de vue cytochimique.

Les fibres axiales des $m v$ ont été étudiées par Milimangton et Finean (I962) et par KJAERHEim ( I $^{6} 3$ ). Le rôle éventuel de ces éléments de rigidité dans les phénomènes d'absorption n'a pas encore été déterminé. Le prolongement de ces fibres dans le cytoplasme sub-apical ("racines " des $\mathrm{mv}$ ) semble être constant; en effet, si parfois, sur une coupe approximativement parallèle au grand axe de la cellule, on constate leur absence, ceci n'est dî qu'à l'incidence inadéquate de la coupe ; sur des sections perpendiculaires à l'axe de la cellule, ces fibres se retrouvent aussi bien pour les cellules situées au sommet que pour celles de la base des villosités. Ces fibres apparaissent parfois formées d'une succession d'éléments sphériques; leur ultrastructure reste à préciser.

Nous avons trouvé une répartition homogène des mitochondries dans la $\mathrm{CE}$ différenciée. Cependant, ZETTERQVisT (I956), PALAY et KARLIN (I959), chez la Souris et le Rat, ont signalé une densité supérieure dans le cytoplasme supra-nucléaire. La structure des mitochondries de la CE du Porc ne diffère pas de celle des cellules 
du même type déjà décrite chez d'autres espèces par ZETTERQvisT, puis PALAY et KARLIN, et semble correspondre aux descriptions relatives à d'autres types cellulaires (PALADE, I953 ; SJÖSTRAND, I963 c). L'épaisseur de la membrane des mitochondries est bien inférieure à celle de la membrane plasmique, comme cela a été constaté par SJöstrand (Ig63 $c, d)$.

Il ne nous semble pas possible de dire, pour aucune région de la CE d'aucun animal étudié, âgé de plus de 3 jours, que l'orientation des mitochondries soit prédominante suivant le grand axe de la cellule, comme cela a été trouvé par certains auteurs chez d'autres espèces (PALAY et KARLIN, I959).

Le réticulum endoplasmique de la CE de l'intestin grêle du Porc présente, comme nous l'avons décrit, une morphologie très variable, suivant les conditions physiologiques ; son rôle n'est qu'incomplètement connu ; cependant, il semble prendre une part importante au transport des graisses ingérées, après leur franchissement de la membrane plasmique (PALAY, I960; VODOVAR et FLÉCHON, I964), transport au cours duquel ont lieu des transformations chimiques (estérifications notamment) et physiques (édifications de chylomicrons). Le réticulum assure un lien continu et permanent entre la membrane du noyau et 1'endoplasme (PALADE, I956 ; EPSTEIN, I957; WATSON, I959; WHALEY, I959) ; il nous paraît le faire également entre la membrane plasmique libre des espaces intermicrovilleux et l'endoplasme (cf. aussi PALAY et REVEL, I964), d'une part, et entre ce dernier et les espaces intercellulaires, d'autre part, ceci sans discontinuité. Entre le fond des espaces intermicrovilleux et le cytoplasme sub-apical, on trouve surtout des tubules et des vésicules de REL. Si la continuité de ces tubules et des vésicules avec le réseau de RER, parallèle à la surface de la cellule et situé dans le cytoplasme supra-nucléaire, semble évidente, leur liaison avec la membrane plasmique libre l'est beaucoup moins, sauf pour les animaux très jeunes. De même, si le débouché du réticulum dans 1'espace intercellulaire apparaît comme indiscutable sur de nombreuses électromicrographies, on ignore, à 1'heure actuelle, comment s'effectue la jonction entre ce réticulum endoplasmique et la membrane plasmique latérale. De même, la continuité du réticulum endoplasmique entre les espaces intermicrovilleux et intercellulaires, à travers le cytoplasme de la cellule épithéliale absorbante, sauf pour les tout jeunes porcelets, nécessite une étude plus approfondie.

Des précisions seront, nous semble-t-il, plus facilement obtenues en étudiant des tissus prélevés au moment du transport des graisses à travers les cellules, à condition, évidemment, de ne pas éliminer ces graisses au cours de la préparation des tissus, ce qui constitue le but de nos travaux en cours.

Rę̧u pour publication en novembre 1965.

\author{
SUMMARY
}

THE INTESTINAL ABSORPTIVE EPITHELIAL CFLLS IN THE PIG, ULTRASTRUCTURE

The absorption accomplishes through the whole epithelial cells (EC) that line the villifolds of the intestine of the Pig (VoDovar et al., 1965), but, in normal physiological conditions, the absorptive power changes according to the age of the animal and particularly to the place of cells on villus. 
The $\mathrm{EC}$ of piglet at birth has no absorptive function yet, and its nucleus is in reversed position. It differs from the EC cut off older animals : we find a different distribution of cytoplasmic elements (mitochondria, endoplasmic reticulum) and lipoproteic inclusions before any absorption of food.

I'ltrastructure quickly alters soon after birth, particularly during the first $3^{6}$ hours. The alterations finish off about 3 days after birth, what seems to correspond to a complete renewal of the intestinal epithelium (VoDovar, not published).

With certain exceptions, cell division takes place in the crypts of mucosa. The cells move up from lower part to the top of villi where they shed and fall in the intestinal lumen. This shifting of cells is produced by sliding on the "basal " membrane. The cells resulting from division do not differ from others, but in the course of shifting their structures change as if they adapted to their absorptive functions. We observed progressive diminution of cross-sections of the cells and lengthening of microvilli - always in constant number. The effect of this change is an increase of the area of exchange with the intestinal medium, and consequently of the absorptive power of cells as they move up the villi.

The epithelial cells of Pig are distinguished by a considerable endoplasmic reticulum. We studied its distribution and orientation.

All this was observed at different levels of villi, that is to say different stages of maturation. This study on the absorptive epithelial cell, the structure of which is closely related to its function, will give way to a better analysis of absorption phenomena, the study of which has been addressed to at the sane time.

\section{RÉFÉRENCES BIBLIOGRAPHIQUES}

Ashwortil G. T., Chears W. C., Jr., Sanders E., Pearce M. B., ig6r. Non tropical sprue. Arch.Path., 71, I3-19.

BAKER J. R., I942. The free border of the intestinal cell of vertebrates. Quart. J. micr. Sci., 84, 73-103.

BAKER J. R., I95I. The absorption of lipoid by the intestinal epithelium of the mouse. Quart. J. micr. Sci., 92, 79-86.

Brettarer J., Steinacir S., 1857. Entersuchungen über das Cylinderepithelium der Darmzotten und seine Bezichung zur lettresorption. S. B. Akad. Wiss. Wien, 23, 303-3 r3.

Browx A. L., ig62. Microvilli of the human jejunal epithelial cell. J. Cell Biol., 12, 623-627.

Crenimer B., Shorter R. G., Bayforth J., ig6I. The turnover and shedding of epithelial cells. I. The turnover in the gastro-intestinal tract. Giut, 2 , г Io-1 I6.

Daltox A. J., Kahler II., Stribrici M. J., Lloyt B., 1950. Finer structure of hepatic, intestinal and renal cells of the mouse as revealed by the electron microscope. J. nat. Cancer Inst., 11, 439-46r.

Dattox A. J., г95. Lilectron micrography of epithelial cells of the gastro-intestinal tract and pancreas. Amer. J. Anal., 89, 109-134.

Daltox A. J., igor. Golgi apparatus and secretion gramuls. In Bracietet J., Mirsky A. L., The Cell, vol. 2, too3-619, Academic l'ress., New York.

EPsterx M. A., 1957. The fine structure of the cells in mouse sarcoma 37 ascitic fluids. J. biophys. biochem. Cytol., 3, $5657-576$.

Favarger P., 1959. L'absorption intestinale et l'utilisation des graisses. 1. Symp. Mainz 1957. In Wiss. Verófftl. Dtsch. Ges. Ernähring, 59-8\&, Dictrich Steinkopff, Darmstadt.

Frazer A. C., rg6. The morphology of fat absorption : questions and discussion. In Meng H. C., Proceedings of an international sympositum on lipit Iransport, 50, Charles C. Thomas, Springfield.

Funke 0., i\$56. Beiträge zur Physiologie der Verdaunng. II. Durchgang des liettes durch das Darmepithel. Zool., 7, 315-327.

GRANES D., I964. Vltrastructure of the apical plasma membrane of the intestinal lining cells. Anat. Rec., 148, 373-374.

Granger B., BAKER R. F., 1949. lilectron microscope investigation of the striated border of intestinal epithelium. Anat. Rec., 103, 459 .

GrangER B., Baker R. F., I950. Electron microscope investigation of the striated border of intestinal epithelium. Anat. Rec., $10 y^{-423-440 .}$

GRUBY, Delafond, i $\mathbf{H}_{43}$. Résultats des recherches faites sur l'anatomie et les fonctions des villosités intestinales, l'absorption, la préparation et la composition du chyle dans les animaux. C. R. Acad. Sci., 16, II $194^{-1} 200$.

Haubrich W. S., Watsen J. H., O'Driscoli W., Valentine V., I959. Llectron microscopy of the free

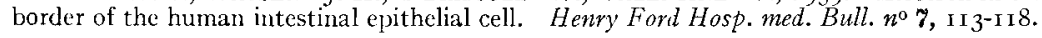

Heideniain R., i 888 . Beiträge zur Ilistologie und Physiologie der Dünndarmschleimhaut. Pfüg. Arch. ges. Physiol., 43, (suppl.), $1-\mathrm{s} 03$. 
Henle J., i84i. Allgemeine Anatomie, 239, Leipzirr. Citć par Baker J. R., I9+2.

KJAERHEIM A., 1963 . The fine structure of the microvilli on principal epithelial cells of mouse jejunum after freeze substitution. J. ullrastruct. Res., 9, 393-394.

KöLIjer A., 1855 . Nachweis eines besonderen Baues der Cylinderzellen des Dünndarms, der zur Fettresorption in lBezug zu stehen scheint. Verh. phys. med. ges. Würsb., 6, 253 .

Kusindo H., ig6r. A styrene methacrylate resin embedding method for ultrathin sectioning. I. Electromicrose, 10, 16-I9.

Leblond C. P., Stevens C. I., I948. The constant renewal of the intestinal epithelium in the albino rat. Anat. Rec.,.100, 357-37I.

Mattison A. G. M., Karlsson B. W., 1964. Sub-light microscopical changes in epithelial cells of the intestine of the pislet reared with and without colostrum. Third Ifuropean Regional Conference on Electron Microscopy, Prague. $433^{1-4}, 32$.

Mc NabB J. D., I964. Filaments and microtubules in intestinal absorptive cells. 1nal. Rec., 148, 310-31 I.

Millington P. F.. FineAN 'T. 13., io62. Electron micrescepe studies of the structure of the microoilli on principal epithelial cells of rat jejunum after treatment in hypo- and hypertonic saline. J. Cell. Biol, 14, $125^{-1} 39$.

Milington P. F., Finean 'T. B3., I963. Studies on the structural integrity of the brush border of rat intestinal epithelial cells. In Frazer A. C., Biochemical problens of lipids, Vol. 1, 116-120, Elsevier Publishing Company, Amsterdam, London, New Fork.

Padane G. E., 1953. An electron microscope study of the mitochondrial structure. J. I1islochen. Cytorhem., 1, I88-193.

PALAde G. F., I956. The endoplasmic reticulumt. J. biophy's. biochem. (vilol., 2, (suppl.) 85-08.

PALAY S. L., KARLIN L. J., 1959. An electron microscopic study of the intestinal villus. I. The fasting animal. J. biophys, biochem. Cylol., 5, 363-371.

PALAY S. L., ig6o. On the appearence of absorbed fat droplets in the nuclear envelope. J. biophys. biochem. Cytol., 7 , 391-392.

Palay S. I., Revei, J. P., I964. The morphology of fat absorption. In MeNc; II. C., Proceedings of an international symposium on lipid transport $33-69$, Charles C. Thomas, Springfield.

Patzelt V., T936. Der Darm. In Von MollendorfF W.. Handbuch der mikroskofischen Analomie des Menschen, vol. 5, Barcimane W., Ileiss R., Leiner J., Patzeli V., l'Lexk II., Verdauungsapparat, $3^{\circ}$ partie Ziihne, Darm Atmungsapparat, I- 4 4. Springer Verlag, Berlin.

RoBertson J. D., I959. The ultrastructure of cell membranes and their derivatives. In The structure and function of subcellular components no $\mathrm{i} 6,3-43$. University Press, Cambriclge.

Robertson J. D., ig6i. The unit membrane. In Boyi J. D., Jonssox If. K., Lever J. D., Electron Microscopy in Inalomy, p. 74-99, Arnold, London.

Sjöstravi) li. S., Zetrerovist A., 1955. Structure of intestinal mucosa. In Hoj.man R. T., LundBerg W. O., Malkin T. Progress in the chemistry of lats and other lifils, vol. 3, 388-393. Pergamon Press, London, New York.

SJöstravi) F. S., 1959. The molecular architecture of the plasma membrane. J. ultrastruat. Res., 3, $239^{-240 .}$

SJöstranis F.S., $\mathrm{Ig}^{6} 3 \mathrm{a}$. The fine structure of the columnar epithelium of the mouse intestine with special reference to fat absorption. In liRAZER A. C., Biochemical problems of lipids, vol, 1, 9I-I 5, Iisevier Publishing Company, Amsterdam, London, New York.

Sjöstrand F.S., $\mathrm{I}_{93} \mathrm{~b}$. The ultrastructure of the plasma membrane of columnar cells of the mouse intestine. J. ultrastruct. Res, 8, 5 I $7-541$.

SiöSTRAND F. S., $\mathrm{rg}_{93} \mathrm{c}$. A new ultrastructural element of the membrames in mitochondria and of some cytoplasmic membranes. J. ullrasinuct. Res., 9, 340-36r.

SjöstrRand F. S., I 963 \%. A comparison of plasma membrane, cytomembranes, and mitochondrial membrane elements with respect to ultrastructural features. J. ultrastruct. Res., 9, 56I-58I.

Taylor A. B., Adamstone F. B., I964. Ultrastructural changes in epithelial cells of crypts and villi of jejunum of the rat. Anat. Rec., 148, 344 .

Tilanioffer L., I93I. Untersuchungen mit Mikrodissektion über die Kutikula und Protoplasmastruktur der Darmepithclzellen. Arch. exp. Zellforsch., 11, 293-299.

Vomovar N., I964. Intestin grele du porc. II. Structure histologique des parois et plus particulièrement de la tunique muqueuse en fonction de l'âge de l'animal. Ann. Biol. anim. Bioch. Biophys., 4, II $3^{-1} 39^{.}$

Vodovar N., Fléchon J. E., I964. Le réticulum endoplasmique des cellules épithéliales, une voie de l'absorption intestinale (note préliminaire). Ann. Biol. anim. Bioch. Biophys., 4, I39-208.

Vodovar N., Thievlin C., Pihet A., Flanzy J., I965. Lieu d'absorption des graisses dans l'intestin grêle du Porc. Ann. Biol. anim. Bioch. Biophys., 5, 249-265.

Watson M. L., I959. Further observation on the nuclear envelope of the aninal cell. J. biophys. biochem. Cylol., 6, 147 -162.

Weiss J. M., I955 $a$. The role of the Golgi complex in fat absorption as studied with the electron microscope with observations on the cytology of duodenal absorptive cells. J. exper. Med., 102, 775-782.

WEISS J. M., I955. $b$ Mitochondrial changes induced by potassium and scdium in the duodenal absorptive cell as studied with the electron microscope. J. exper. Med., 102, 78,3-788. 
Welcker H., 1857. Bemerkungen zur Mikrographie. II. Ueber elastiche Faser, Muskelfaser und Darmepithel. Z. rationnelle Med., Neue Folge, 8, 225-241.

Wiraley W. G., I959. Dynamics of cell ultrastructure in development and growth. Science, 1425-1426.

ZETTERQVIST H., I956. The ulirasiructural organization of the columnar absorbing cells of the mouse jejunum. Thesis, Karolinska Institutet, Stockholm. 83 p.

Zimmermann K. W., I 898 . Beiträge zur Kenntnis einiger Drüsen und Epithelien. Arch. mikr. Anat., 52, 5,52-706. 
ABRÉVATIONS EMPLOYÉS SUR LES ÉLECTROMICROGRAPHIES

$\mathrm{Ag} \quad=$ appareil de Golgi.

$\mathrm{Ca}=$ couche apicale.

$\mathrm{Chr}=$ chromosomes.

$\mathrm{D}=$ desmosomes.

$\mathrm{E} \mathrm{i}=$ espace intercellulaire.

Eimv = espace intermicrovilleux.

Fmv = fibres des microvillosités.

Icp = inclusion cytoplasmique.

$\operatorname{Imp}=$ invagination de la membrane plasmique.

jc $=$ jonction cellulaire.

$\mathrm{I}_{\mathrm{i}}=$ lysosomes.

$\mathrm{M}=$ mitochondries.

$\mathrm{mb}=$ " basale " (membrane séparant la couche épithéliale du stroma).

$\mathrm{mp}=$ membrane plasmique.

$\mathrm{mv}=$ microvillosité.

$\mathrm{N}=$ noyau.

$\mathrm{Pmp}=$ plissement (interdigitation) de la membrane plasmique.

$\mathrm{R}=$ ribosome.

$\mathrm{Ra}=$ racine de la microvillosité.

$\mathrm{RE}$ = réticulum endoplasmique.

$\mathrm{REL}=$ réticulum endoplasmique lisse.

$\mathrm{RER}=$ réticulum endoplasmique granuleux.

$\mathrm{Rmp}=$ jonction du réticulum endoplasmique avec la membrane plasmique.

tb $=$ bandelette obturante.

$\mathrm{V}=$ vésicules. 
FIG. A. - Villosilés de l'intestin grèle du porc a) sommets des villosités (partie distale)

b) côtés des villosités (partie médiane)

c) bases des villosités (partie proximale)

d) régrions des cryptes 


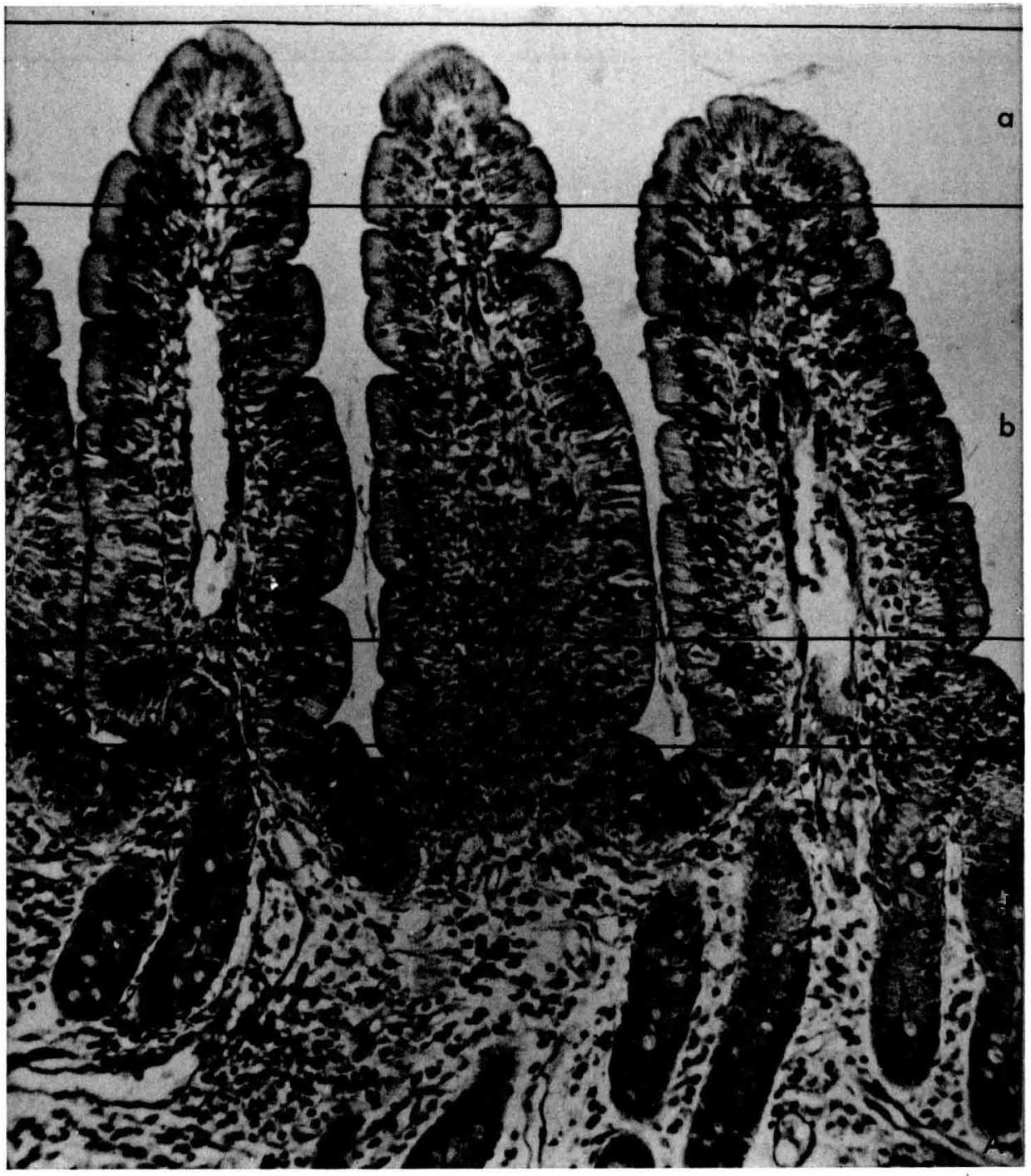




\section{FIG. B}

Schéma d'une cellule épithéliale absorbante :

Microvillosités (mv), zone apicale (ca), région supranucléaire $(\mathrm{SN})$, région périnucléaire (PN) et région sub-nucléaire (DN). Fibres de microvillosités (Fmv), espaces intermicrovilleux (Eimv) racines de microvillosité (Ra), bandelette obturante (tb), desmosomes (D), espace intercellulaire (ei), membrane plasmique (mp), jonction de RER avec la membrane plasmique (Rmp) et la "basale " (mb). Plissement (interdigitation) de la membrane plasmique (Pmp). Réticulum endoplasmique rugueux (RER), mitochondries $(\mathrm{M})$, noyau $(\mathrm{N})$. 


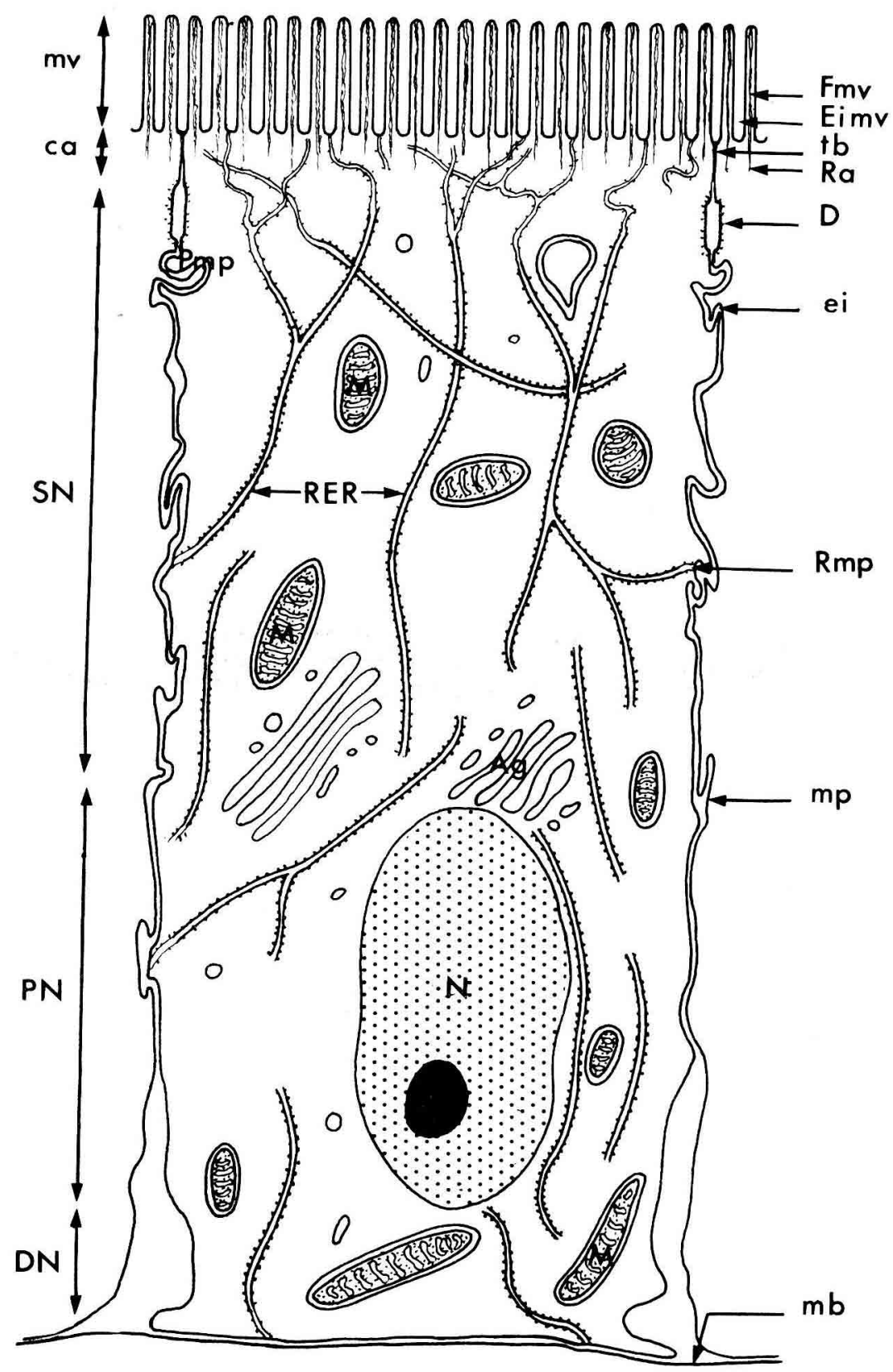

(B) 
FIG. C. - Schéma dune microvillosité de la cellute épithéliale absorbante Membrane plasmique (mp) avec ses 3 couches $a, b$, c. Fibres de la microvillosite (Fmv). Fond d'espace intermicrovilleux (Eimv). Invaginations de la membraue plasmique (Imp). Racines de microvillosité (Ra). 


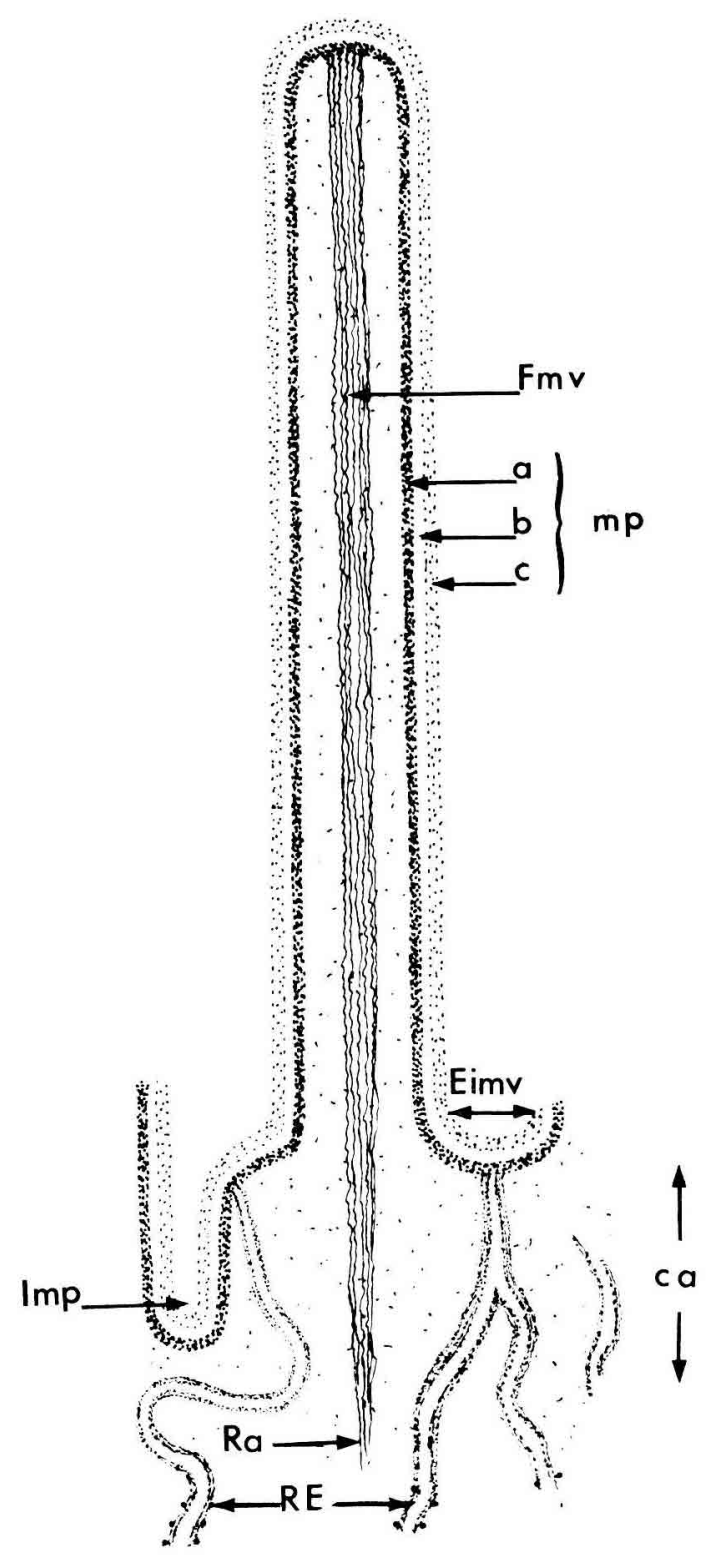

(C) 
JílG. I. - Cellule épithéliale de l'intestin grêle du porcelet, prélevée immédiatement après la naissance (sans ingestion de nourriture), sur la partie distale de la villosité, dans le premier tiers du jéjunum.iléon

Présence de nombreuses inclusions lipidiques (Icp) de tailles différentes. Microvillosités (mv) régulières, zone apicale bien distincte (ca), mais non différenciée, nombreux tubules (RE), noyau (N) près de la zone apicale. Les cellules sont bien liées au niveau apical ou l'on trouve de nombreuses interdigitations (Pmp). Sur le reste du pourtour, les espaces intercellulaires (ei) sont trés importants et remplis de substances. Les mitochondries (M) sont groupées dans la partie basale de la cellule. ( $\mathrm{SSO}_{4} 2$ p. 100, Métacrylate, Acétate d'U, $\times 4500$ ). 


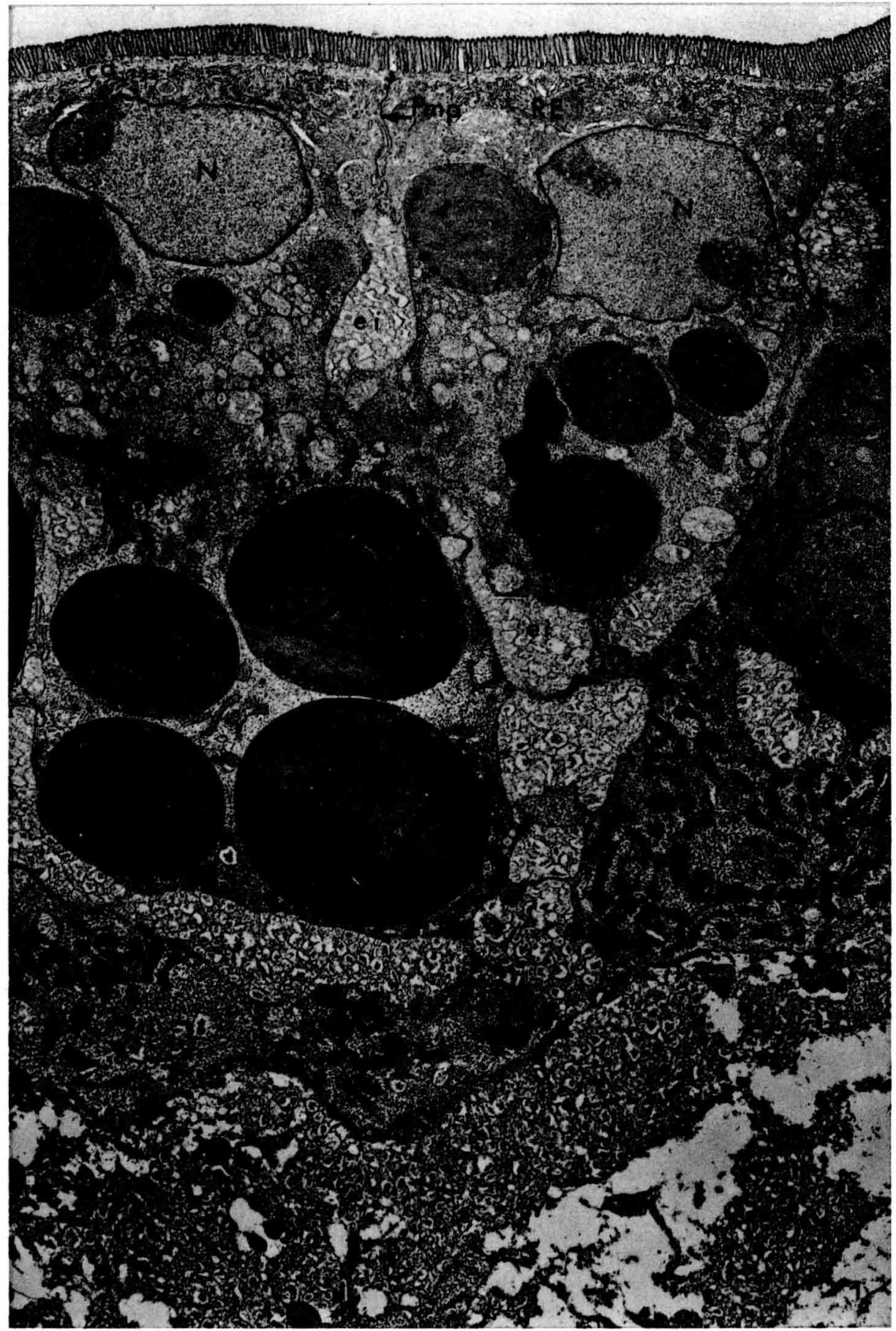


FIG. 2

Cellule épithéliale absorbante de l'intestin grêle du porcelet prélevée sur la partie distale de la villosité, dans le premier tiers du jéjunum-iléon, $18 \mathrm{~h}$ après la naissance et $6 \mathrm{~h}$ après ingestion de colostrum.

Microvillosité (mv), bandelette obturante (tb), zone apicale (ca), avec des tubules (RE), plus large et plus différenciée que sur la figure 1. Très nombreuses interdigitations latérales ('mp). Le noyau (N) est encore dans la région sub-apicale. Les mitochondries (M), sans être entièrement réparties dans le cytoplasme, ont migré, en partie, au-dessus du noyau. Le réticulum de forme tubulaire ou vésiculaire se situe surtout dans la partie basale de la cellule (RER). La face inférieure des cellules est plane, la "basale " (mb) est parallèle à la membrane plasmique.

$\left(\mathrm{OsO}_{4} 1\right.$ p. 100, méthacrylate, acétate d'U, sandwich, $\left.\times 8000\right)$. 


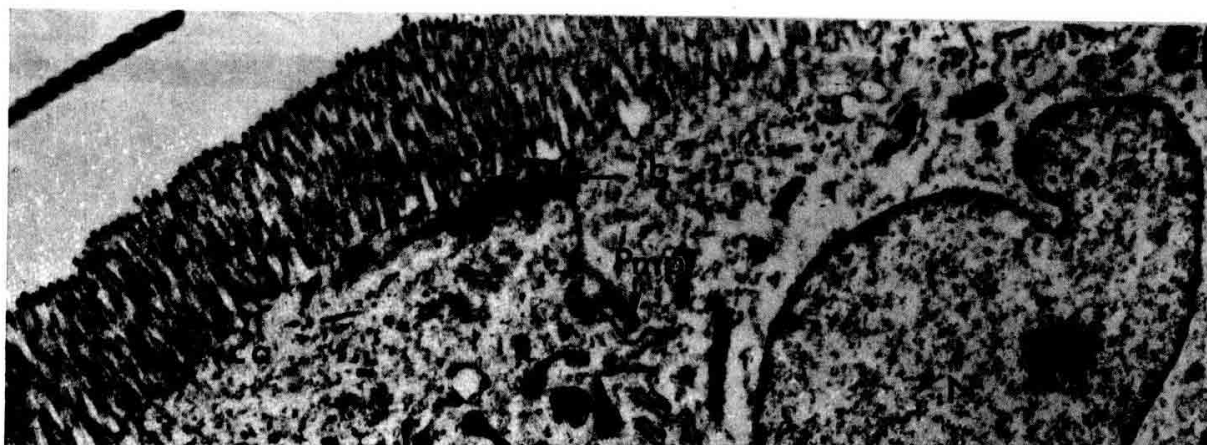

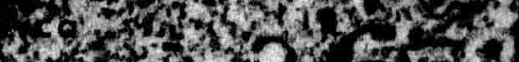

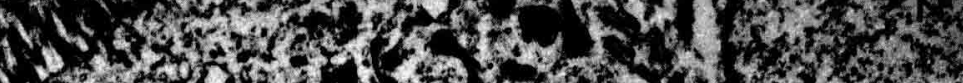
1. Dof 15.

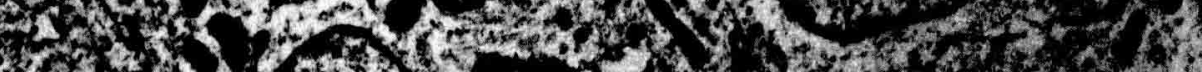

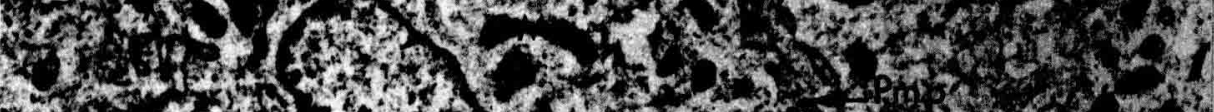

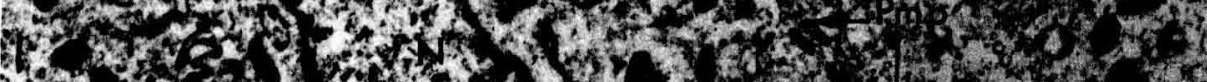

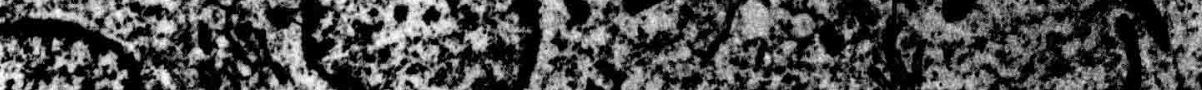
32.5.

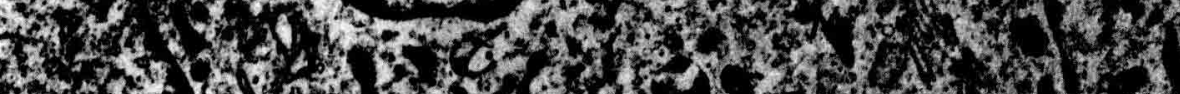
1.

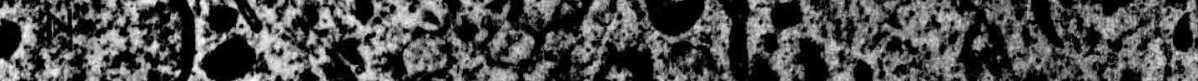

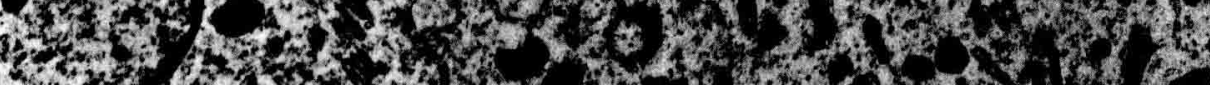

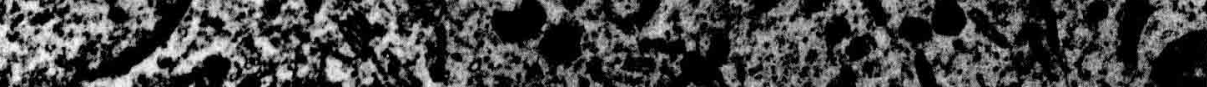
H.

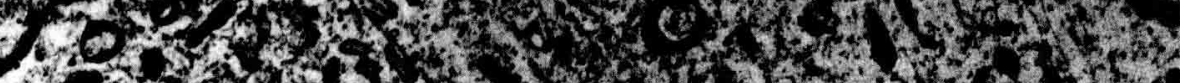

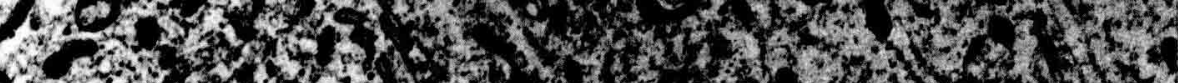

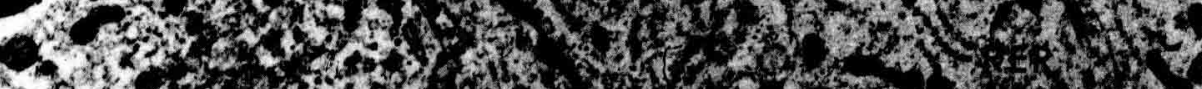

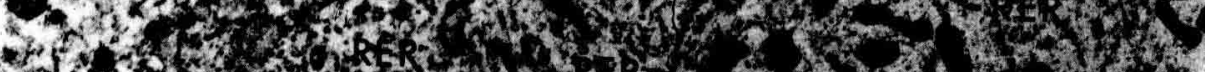

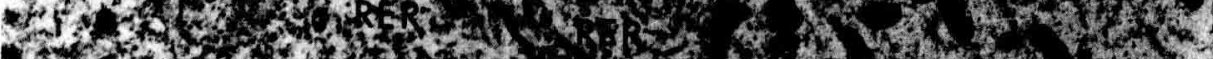

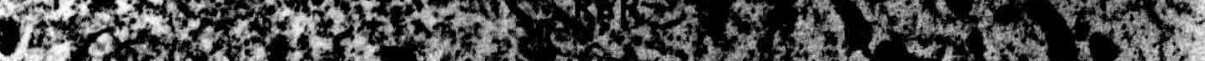

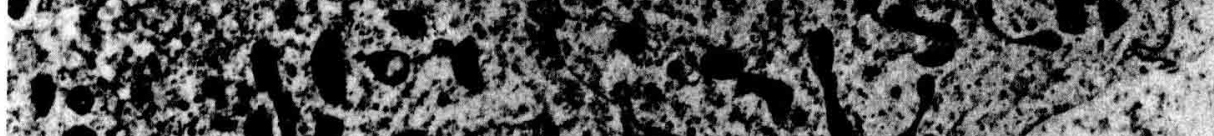

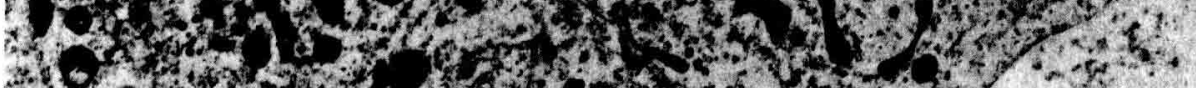

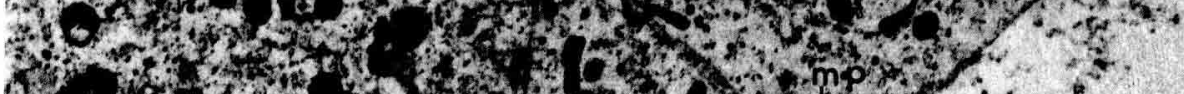

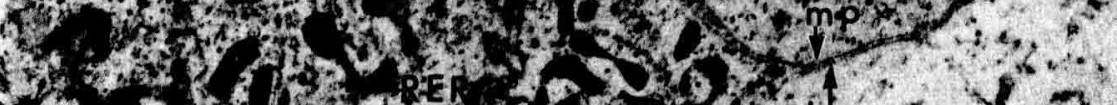

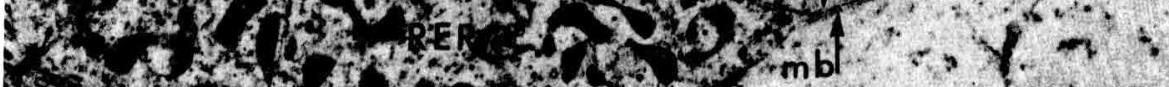

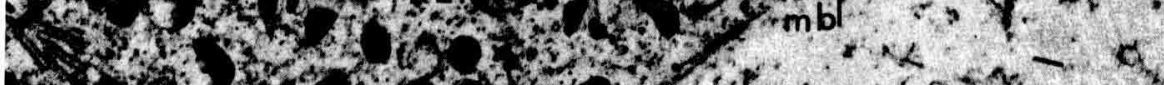

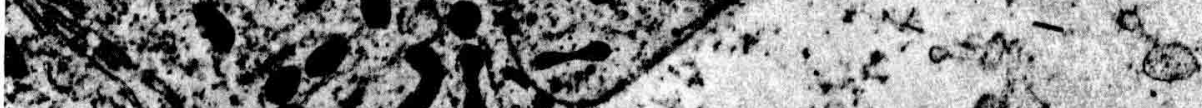

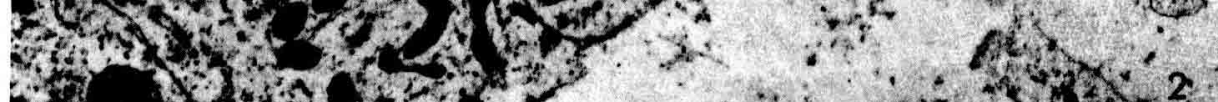

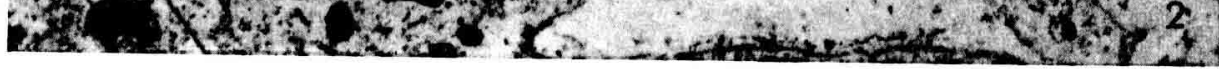




\section{FIG. 3}

Cellule épithéliale absorbante de l'intestin grêle du porc adulte prélevée sur la partie distale de la villosité, dans le premier tiers du jéjunum-iléon, après $18 \mathrm{~h}$ de jeûne.

Microvillosités (mv), couche apicale (ca) d'apparence homogène et pourvue de rares vésicules (V) et de réticulum endoplasmique (RE).

Le réticulum endoplasmique rugueux (RER), disséminé dans le cytoplasme, est plus abondant dans la partie supranucléaire qu'au-dessous du noyan $(\mathrm{N})$. Les mitochondries $(\mathrm{M})$ sont d'orientation variable et assez régulièrement réparties dans le cytoplasme. Les interdigitations de la membrane plasmique latérale (Pmp) sont surtout abondantes dans la partie située au-dessous des desmosomes (D) et sont plus espacées vers la base de la cellule. Vésicules golgiennes $(\mathrm{Ag})$ et noyau $(\mathrm{N})$.

$\left(\mathrm{OsO}_{4}\right.$, méthacrylate, $\left.\times 9000\right)$.

Fia. 4

Jonction des membranes de deux cellules épithéliales adjacentes (tb). Desmosomes (D) qui se succèdent à des niveaux différents; nombreuses fibrilles.

$\left(\mathrm{OsO}_{4}\right.$, méthacrylate, acétate d' $\left.\mathrm{d}, \times 60000\right)$.

FIG. 5

Les membranes des deux cellules épithéliales adjacentes au niveau apical (tb) ne sont pas jointes. Desmosomes (D). Racine des fibres d'une microvillosité (Ra).

$\left(\mathrm{OsO}_{4}\right.$, méthacrylate, acétate d'U, $\left.\times 60000\right)$. 


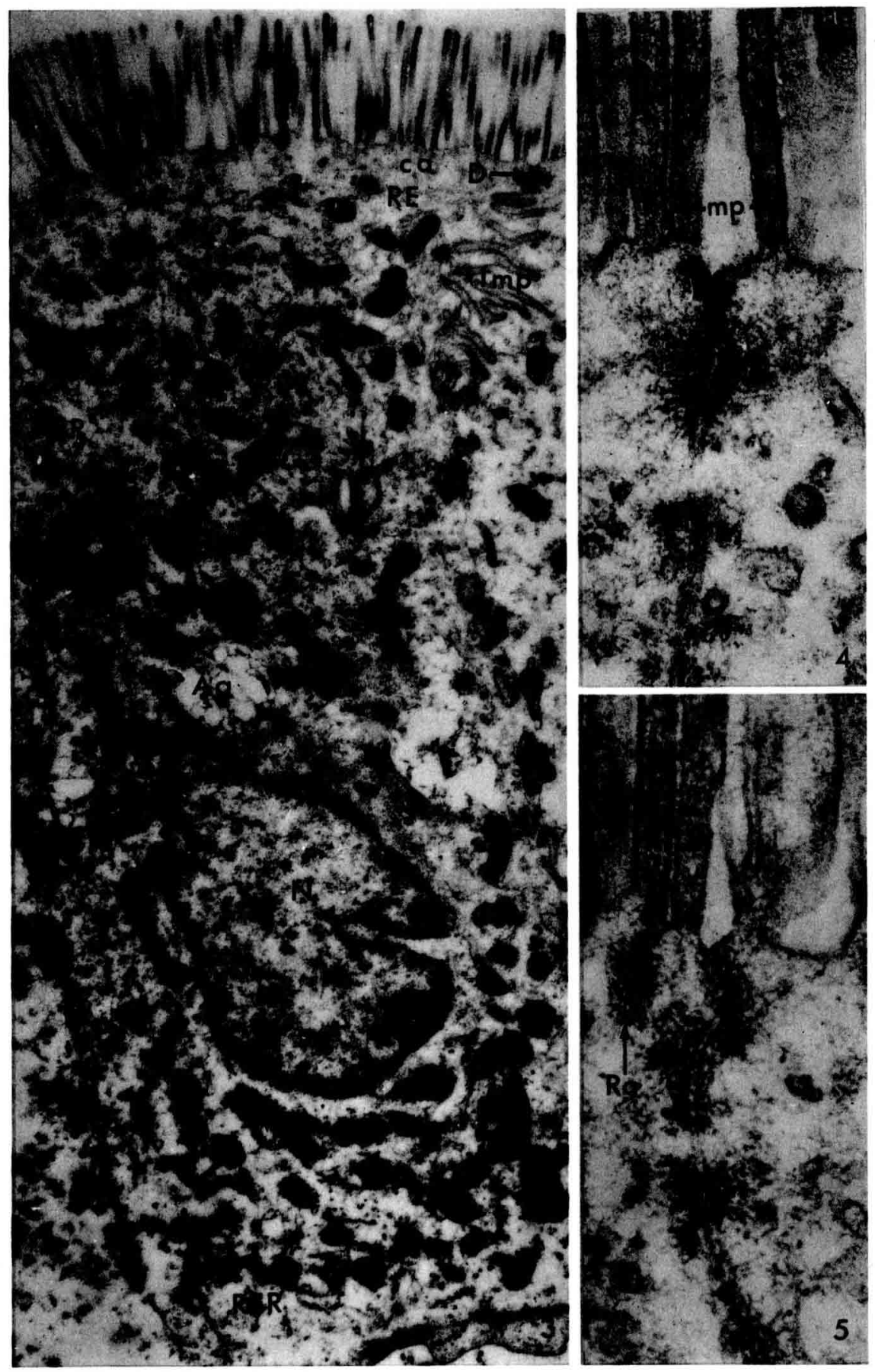


FIg. 6

Vue générale de la partie apicale des cellules épithéliales (dont une en mitose) dans les cryptes de Lieberkühn, prélevées sur le Porc, dans la partie proximale du jéjunum-iléon.

Microvillosités (mv) courtes; les espaces relativement importants entre les microvillosités sont remplis de mucus. Les cellules sont maintenues latéralement entre elles surtout par les desmosomes (D). Peu d'interdigitations entre les membranes plasmiques (mp).

Le réticulum endoplasmique (RE) relativement abondant entoure les chromosomes de la cellule en mitose. La zone apicale est peu précise par rapport aux figures précédentes. Mitochondries (M) réparties au hasard et entremêlées d'éléments à forte densité (lysosomes?).

$\left(\mathrm{OsO}_{4}\right.$, méthacrylate, acétate d'U, sandwich, $\left.\times 24000\right)$, 


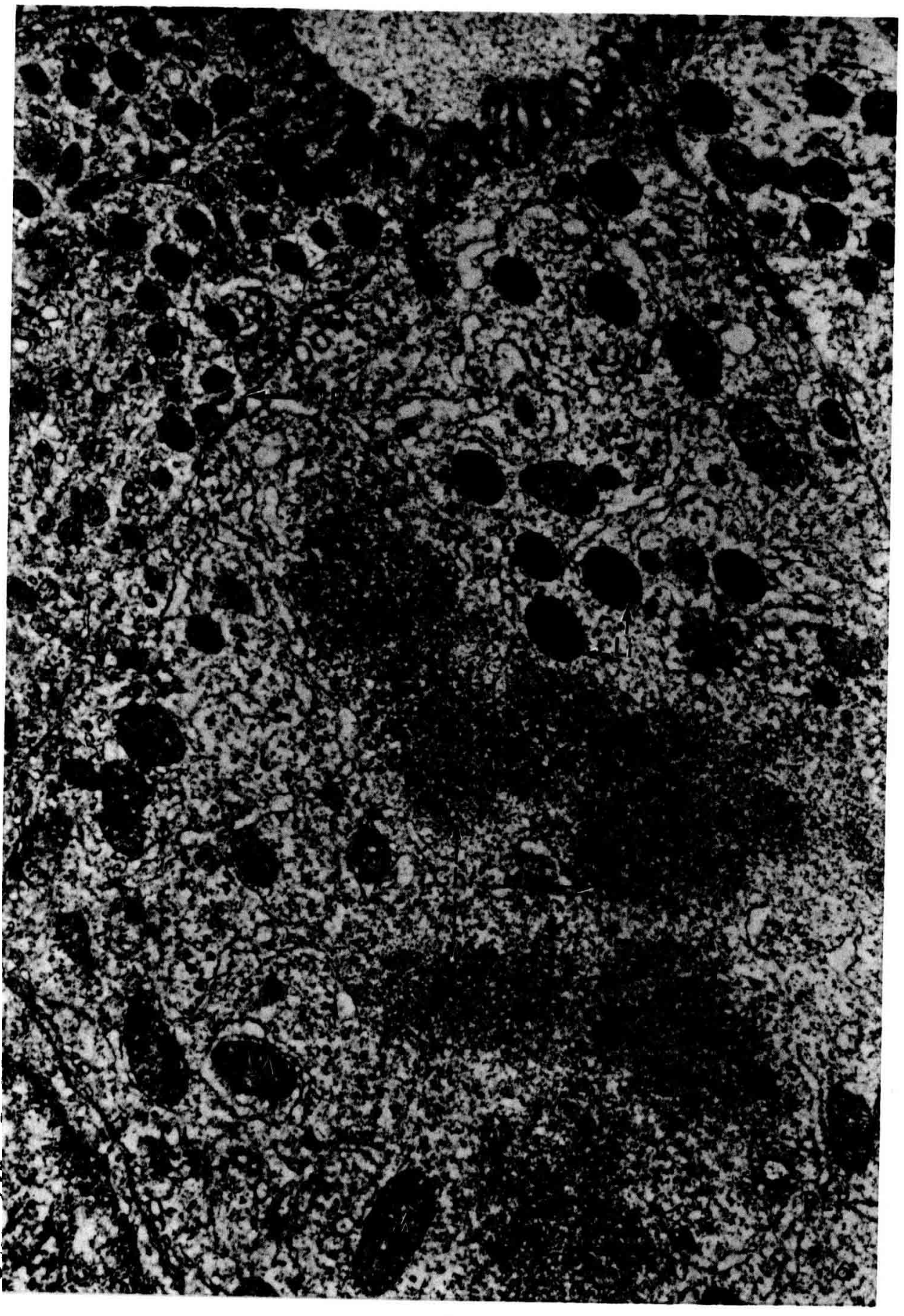


FIG. 7

Microvillosités et zone apicale de deux cellules adjacentes prélevées sur la région intermédiaire de la villosité, dans la partie proximale du jéjunum-iléon.

Les microvillosités (mv). sont plus longues et plus denses et de diamètre (au même grossissement) inférieur à celui de la fig. 6; bandelette obturante (tb) et desmosomes (D). Plissements caractéristiques des membranes plasmiques latérales (P̉mp). Espaces intercellulaires (ei) au sommet d'une interdigitation. La membrane plasmique ( $\mathrm{mp}$ ) semble être en continuité avec le réticulum endoplasmique à différents niveaux ( $\mathrm{Rmp}$ ).

$\left(\mathrm{OsO}_{4} 1\right.$ p. 100, méthacrylate, acétate d'U, $\left.\times 72000\right)$. 


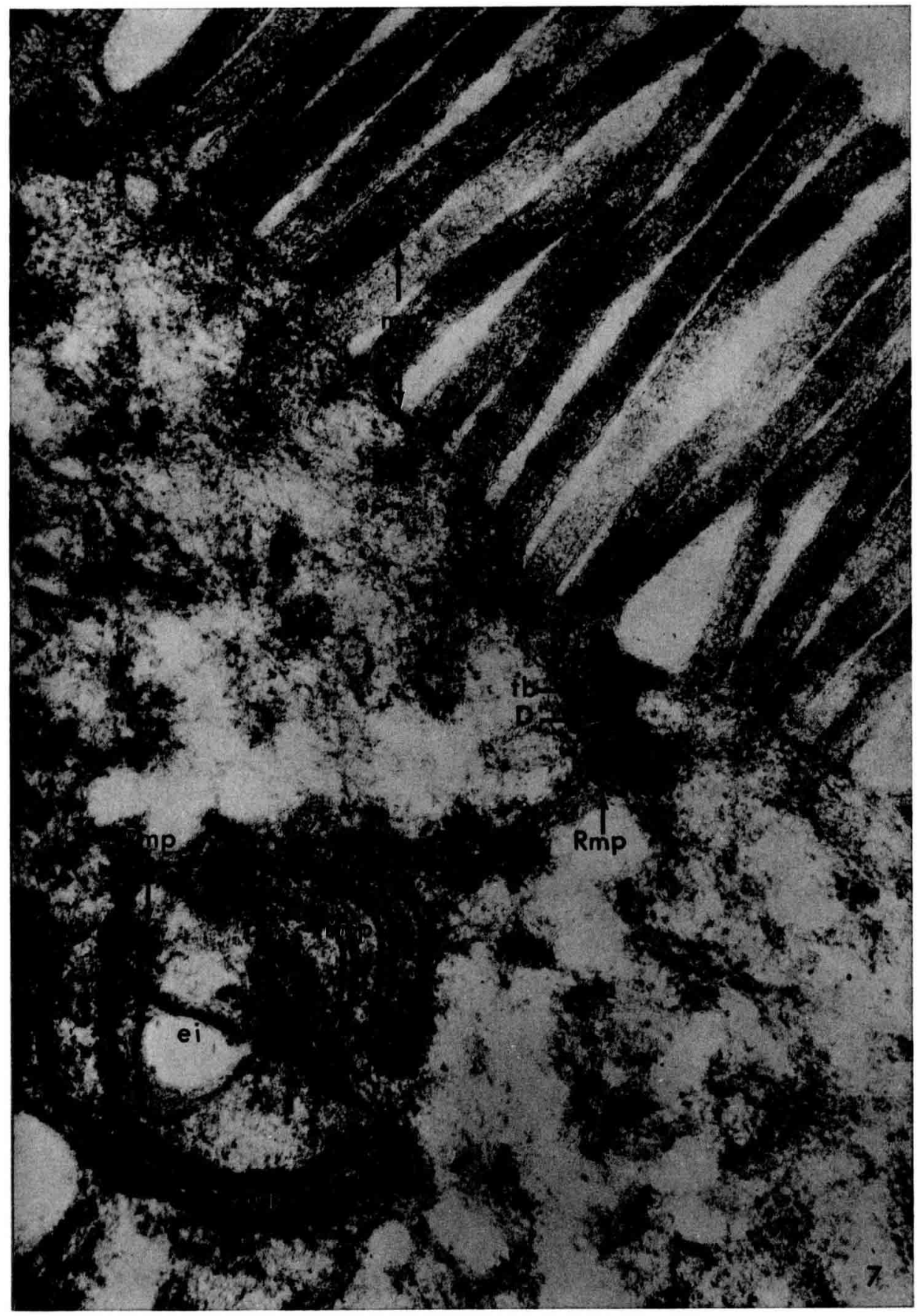


FIG. 8

Microvillosités et couche apicale de la cellule épithéliaje absorbante prélevée sur la partie distale des villosités, dans la partie proximale du jéjunum-iléon.

Microvillosités (mv) très longues. Membrane plasmique libre ( $\mathrm{mp}$ ) apparaissant avec sa structure classique de 3 couches. Espace intermicrovilleux (Eimv). Racine des fibres d'une microvillosité (Ra).

$\left(\mathrm{OsO}_{4} 1\right.$ p. 100, méthacrylate, acétate d'U, $\left.\times 60000\right)$.

FIG. 9

Microvillosités et couche apicale de deux cellules absorbantes prélevées sur la partie proximale de la villosité dans le premier tiers du jéjunum-iléon du Porc.

Les microvillosités ( $\mathrm{mv}$ ) sont courtes avec un diamètre relativement grand. On distingue : 3 couches de la membrane plasmique libre $(\mathrm{mp})$, bandelette obturante (tb) et desmosome (D). La zone apicale (ca) est homogène. A la limite de la zone apicale, le réticulum endoplasmique (RE) est souvent parallèle à la surface libre.

$\left(\mathrm{OsO}_{4}\right.$, méthacrylate, acétate d'U, sandwich $\left.\times 60000\right)$. 

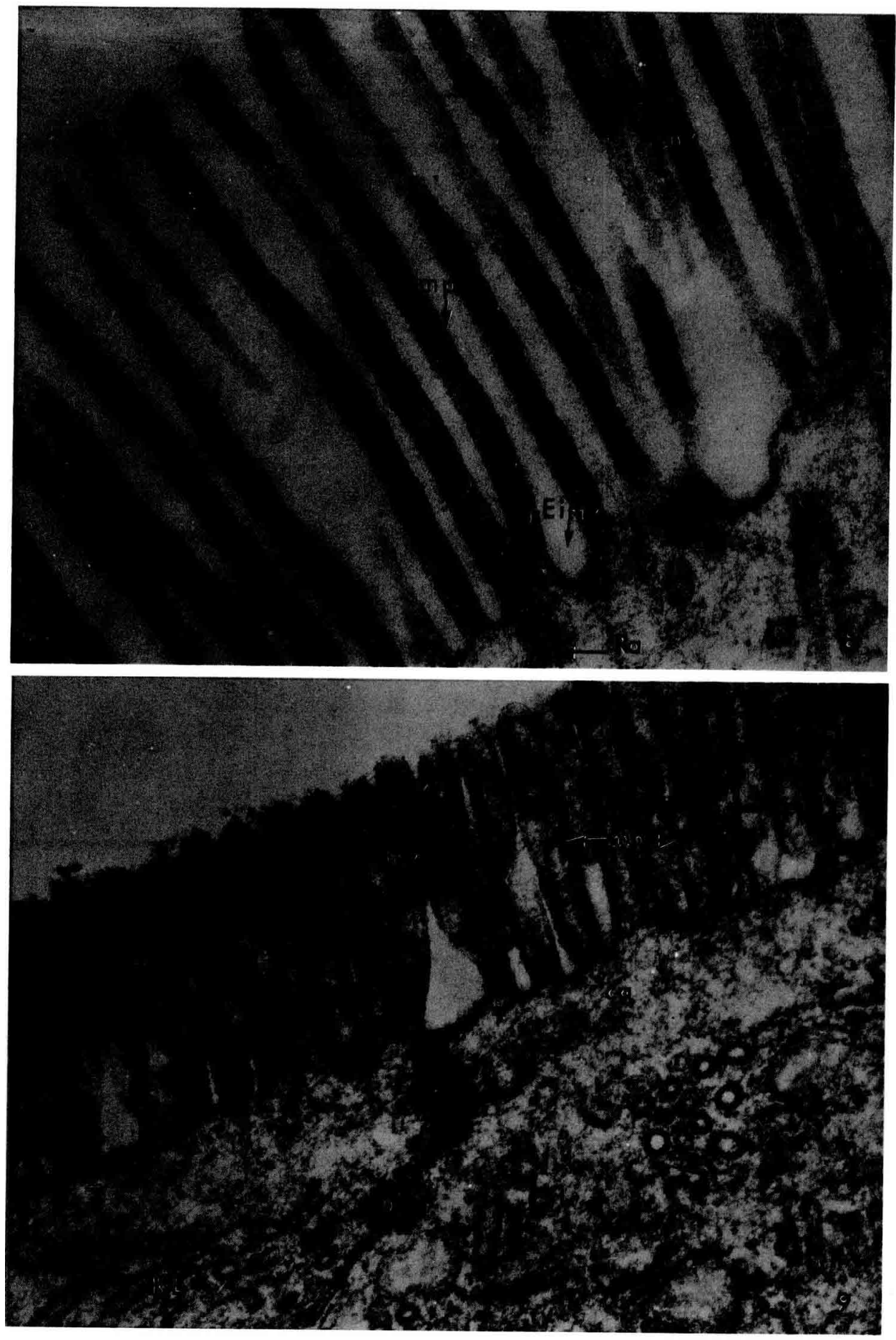
Fig. 10

Microvillosités de la cellule épithéliale absorbante, en coupe perpendiculaire à leur axe, coupées près de leur base, sur la partie distale de la villosité, dans le tiers proximal du jéjunumiléon.

Membrane plasmique libre (mp). Fibres des microvillosités (Fmv). Autour des microvillosités, espaces intermicrovilleux (Eimv).

$\left(\mathrm{OsO}_{4} 1\right.$ p. 100, méthacrylate, sandwich $\left.\times 60000\right)$.

FIG. 11

Partie basale de la cellule épithéliale absorbante.

Membrane plasmique (mp), "basale " (mb) qui sépare la couche épithéliale du stroma, espace intercellulaire (ei), noyau $(\mathrm{N})$, cellule endothéliale (ce).

$\left(\mathrm{OsO}_{4} 2\right.$ p. 100, méthacrylate prépolymérisé, acétate d'U, $\times 30000)$. 


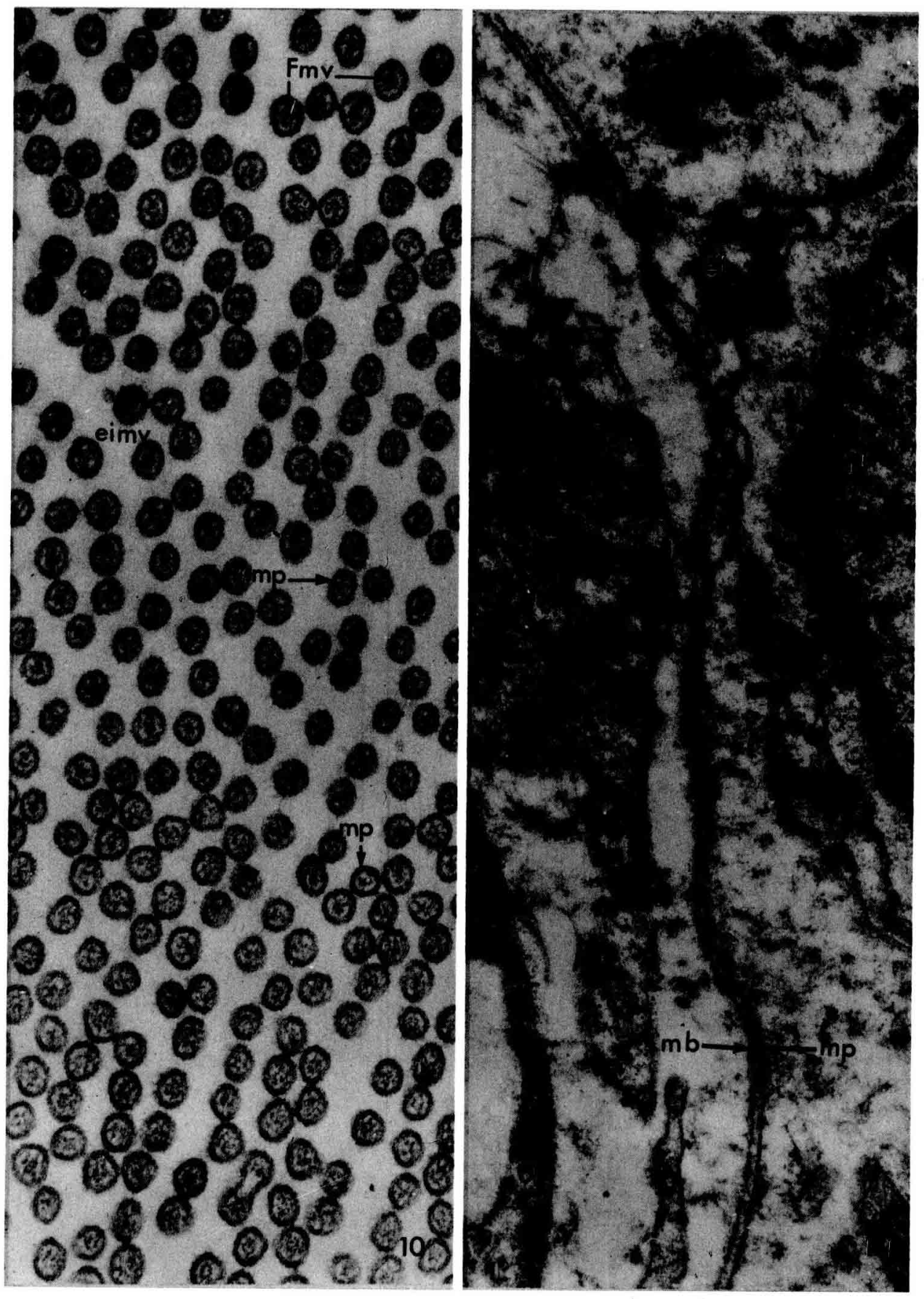


FIG. 12

Membrane plasmique latérale de deux cellules adjacentes. Espaces intercellulaires (ei) très importants, par endroits remplis de substance; fusion probable du réticulum endoplasmique avec la membrane plasmique latérale $(\mathrm{Rmp})$, mitochondries $(\mathrm{M})$, réticulum endoplasmique granuleux (RER) (sections d'aspect tubulaire et circulaire), noyau (N).

$\left(\mathrm{OsO}_{4} 1\right.$ p. 100 , méthacrylate, $\left.\times 60000\right)$. 


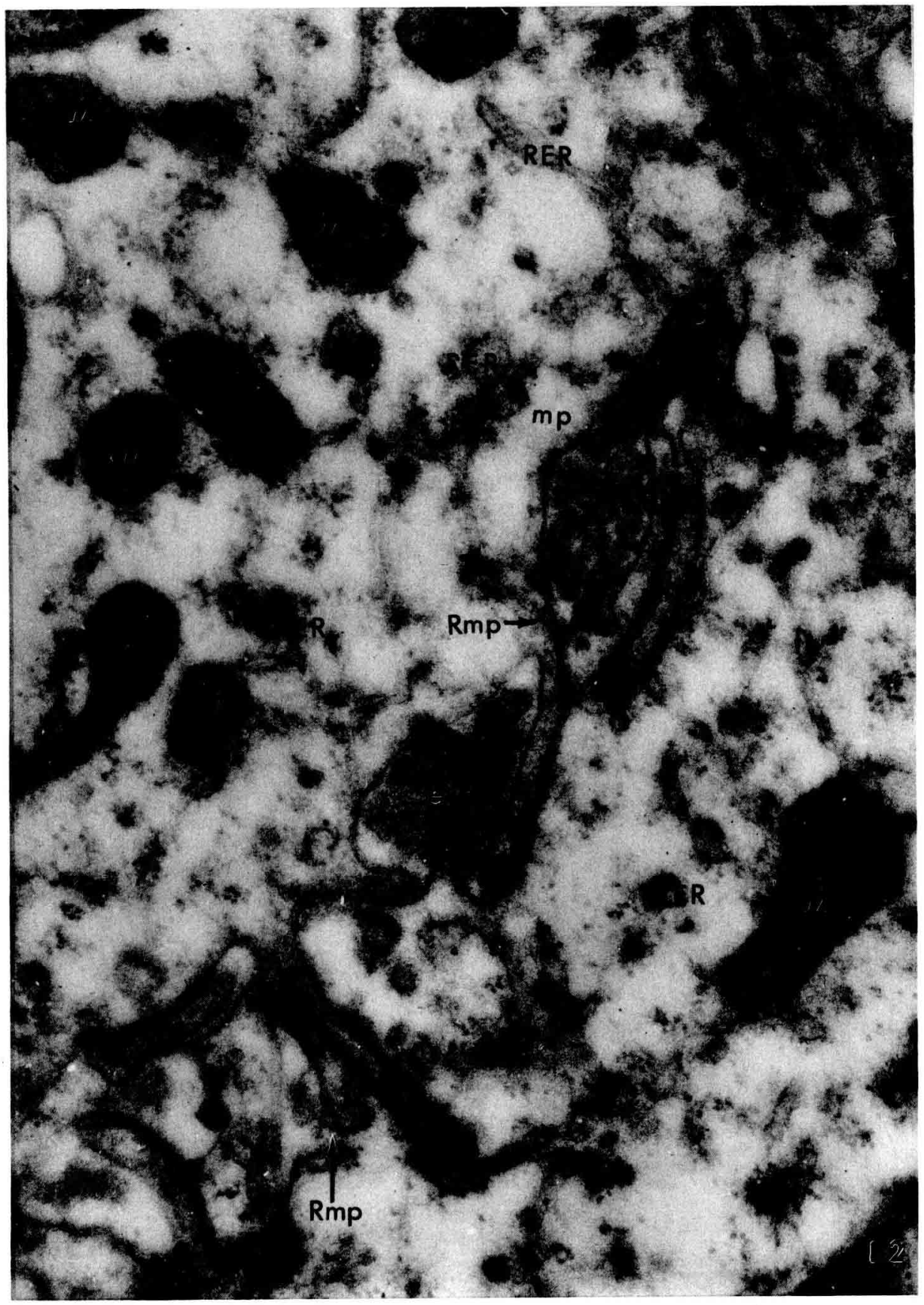

Phototypie Brunissen - Paris 
FIG. 13

Région sub-apicale de la cellule absorbante (coupe parallèle à la surface de la cellule), prélevée sur la partie proximale de l'intestin grêle du porcelet.

Réticulum endoplasmique granuleux (RER) abondant. Mitochondries $(M)$; interdigitations de la membrane plasmique latérale (Pmp); fusion probable entre RER et membrane plasmique (Rmp).

$\left(\mathrm{OsO}_{4} 1\right.$ p. 100, méthacrylate, acétate d'U, $\left.\times 60000\right)$. 


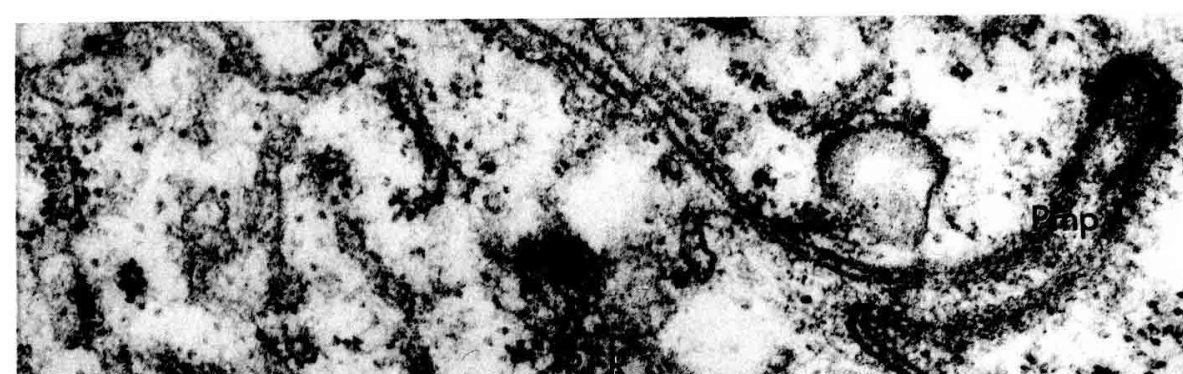
6.

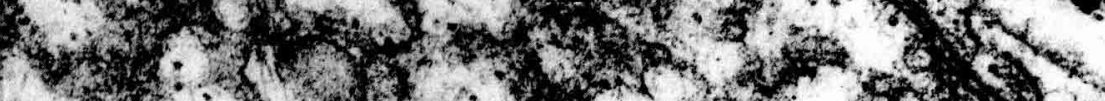

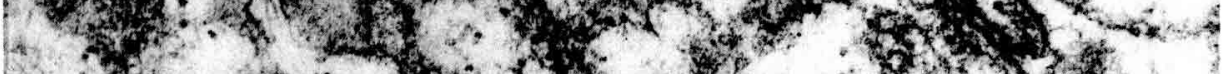

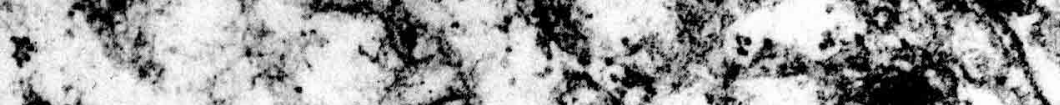

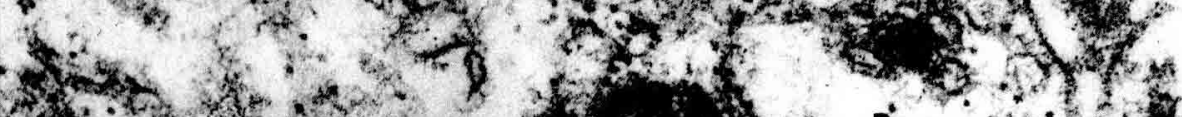

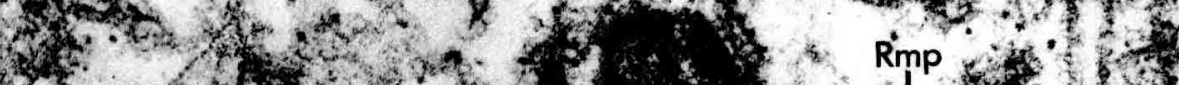

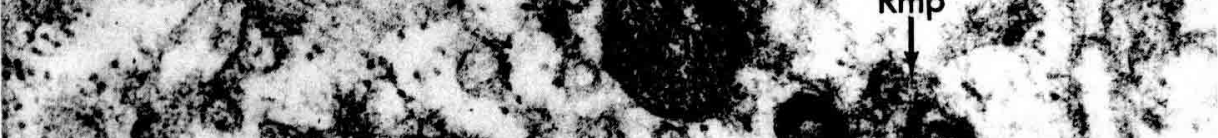

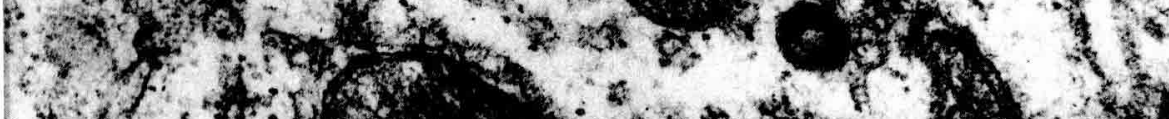

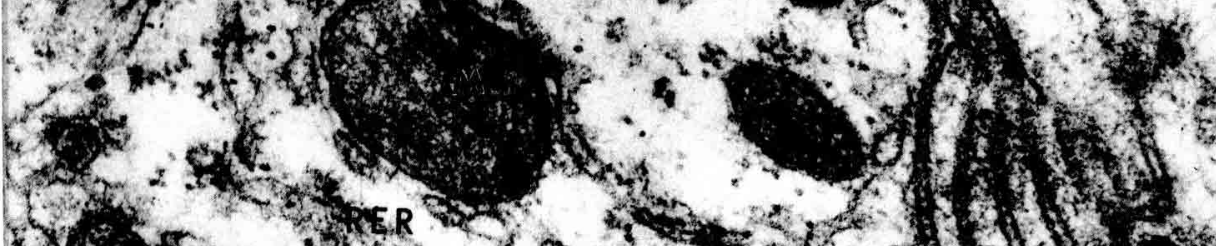

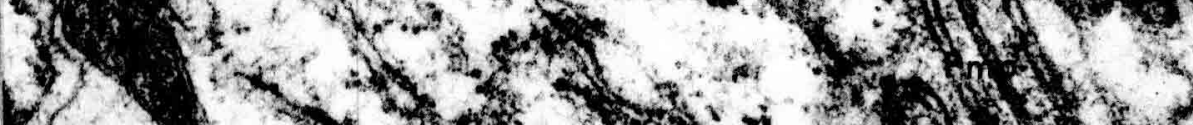

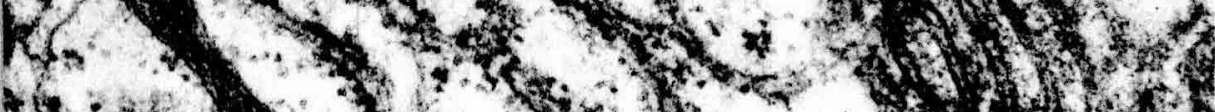

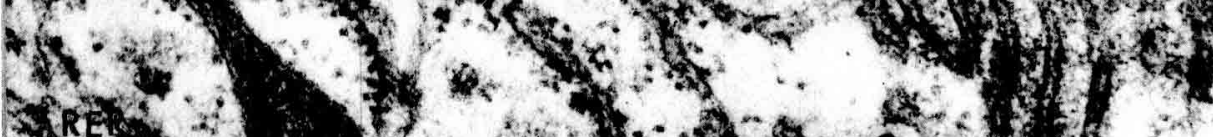

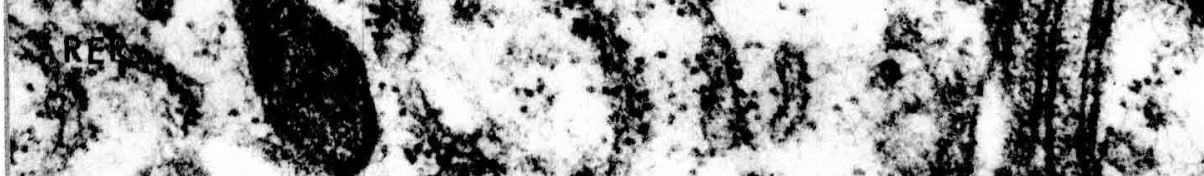

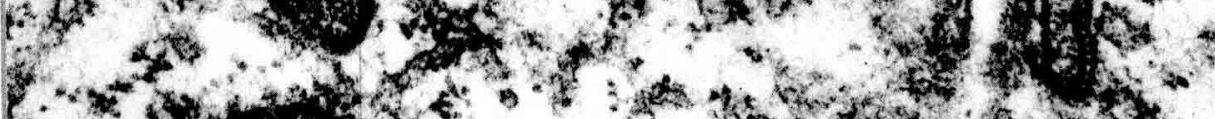

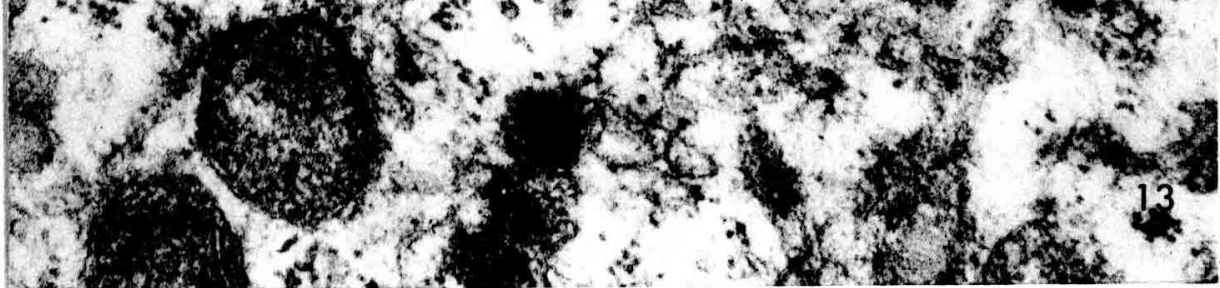

